\title{
Apelin Protects Primary Rat Retinal Pericytes from Chemical Hypoxia-Induced Apoptosis
}

\author{
Li Chen, ${ }^{1,2}$ Yong Tao, ${ }^{1,2}$ Jing Feng, ${ }^{1,2}$ and Yan Rong Jiang ${ }^{1,2}$ \\ ${ }^{1}$ Department of Ophthalmology, People's Hospital, Peking University, 11 Xizhimen South Street, Xicheng District, Beijing 100044, China \\ ${ }^{2}$ Key Laboratory of Vision Loss and Restoration, Beijing Key Laboratory of Diagnosis and Therapy of Retinal and Choroid Diseases, \\ Ministry of Education, 11 Xizhimen South Street, Xicheng District, Beijing 100044, China
}

Correspondence should be addressed to Yan Rong Jiang; drjyr@gmail.com

Received 27 November 2014; Revised 24 January 2015; Accepted 10 February 2015

Academic Editor: Juliana L. Dreyfuss

Copyright (C) $2015 \mathrm{Li}$ Chen et al. This is an open access article distributed under the Creative Commons Attribution License, which permits unrestricted use, distribution, and reproduction in any medium, provided the original work is properly cited.

\begin{abstract}
Pericytes are a population of cells that participate in normal vessel architecture and regulate permeability. Apelin, as the endogenous ligand of G protein-coupled receptor APJ, participates in a number of physiological and pathological processes. To date, the effect of apelin on pericyte is not clear. Our study aimed to investigate the potential protection mechanisms of apelin, with regard to primary rat retinal pericytes under hypoxia. Immunofluorescence staining revealed that pericytes colocalized with APJ in the fibrovascular membranes dissected from proliferative diabetic retinopathy patients. In the in vitro studies, we first demonstrated that the expression of apelin/APJ was upregulated in pericytes under hypoxia, and apelin increased pericytes proliferation and migration. Moreover, knockdown of apelin in pericyte was achieved via lentivirus-mediated RNA interference. After the inhibition of apelin, pericytes proliferation was inhibited significantly in hypoxia culture condition. Furthermore, exogenous recombinant apelin effectively prevented hypoxia-induced apoptosis through downregulating active-caspase 3 expression and increasing the ratio of $\mathrm{B}$ cell lymphoma-2 (Bcl-2)/Bcl-2 associated $\mathrm{X}$ protein (Bax) in pericytes. These results suggest that apelin suppressed hypoxia-induced pericytes injury, which indicated that apelin could be a potential therapeutic target for retinal angiogenic diseases.
\end{abstract}

\section{Introduction}

Pericytes are a population of contractile cells that surround the endothelial cells of microvessel [1]. Genetic studies have shown that pericytes exert multiple effects on the vasculature: they participate in vascular development, maturation, and remodeling, and they also contribute to normal architecture and regulate permeability [1-3]. Retina, a light sensitive layer lining at the back of the eye, has the highest pericyte density around the body [4]. Recently, pericytes have received considerable attention as an active player in the pathological mechanisms of retinal angiogenic diseases, such as diabetic retinopathy (DR) and retinopathy of prematurity (ROP) $[5$, 6]. DR is one of the most common and important chronic microvascular complications in individuals with diabetes mellitus, which will give rise to blindness in uncontrolled conditions. Numerous studies have shown that the primary morphological change in the diabetic retina is the dysfunction and loss of pericytes [7]. The absence of pericytes destabilizes retinal vessels, making them more susceptible to hypoxia-induced sprouting $[7,8]$. Others showed that pericytes are also involved in pathological retinal angiogenesis in a murine model of ROP [9]. Therefore, pericytes are currently under consideration as therapeutic targets for the treatment of retinal angiogenic diseases.

Apelin, a natural ligand for an orphan $\mathrm{G}$ protein-coupled receptor APJ, is widely expressed in various tissues, including brain, heart, lung, kidney, uterus, and ovary $[10,11]$, and is reported to be involved in the regulation of multiple physiological functions [12]. The studies of apelin-deficient mice and Xenopus laevis embryos indicate that apelin is involved in the regulation of vasculogenesis and angiogenesis $[13,14]$. Hara et al. showed that the size of choroidal neovascular membrane lesions was decreased in apelin gene knockout mice [15], and in vitro studies have shown that apelin induced proliferation and migration of vascular smooth muscle cells (VSMCs) and suppresses VSMCs apoptosis induced by serum deprivation via APJ/PI3-K/Akt signaling pathways [16, 17]. In endothelial 
cells apelin also significantly enhanced migration, proliferation, and capillary-like tube formation [18]. Besides, our previous studies showed that apelin significantly enhanced the viability, migration, and proliferation of Müller cells and retinal pigment epithelium (RPE) cells through the pathway of MAPK/Erk and PI3-K/Akt [19-21].

Hypoxia has been widely used as a typical apoptosis insult to a variety of cell types [22]. Hypoxia has been shown to induce rat pancreatic $\beta$-cell apoptosis through $\mathrm{Bcl}-2 / \mathrm{Bax}$ pathway [23]. Previous studies demonstrated that apelin suppressed apoptosis in osteoblastic cell, human osteoblasts, and bone marrow mesenchymal cells through regulation of Bcl-2/Bax via PI3-K pathway [24-26]. Moreover, apelin reduced cytochrome $\mathrm{c}$ release from mitochondria to cytoplasm and activation of caspase 3. These results explained apelin protected cells via various mechanisms.

However, whether apelin has protective effects on rat primary pericytes has not been explored. We propose a putative role for apelin in the viability and apoptosis of pericytes and conducted this study to investigate whether apelin could exert protective effects on primary rat retinal pericytes under hypoxia.

\section{Materials and Methods}

2.1. Reagents. Exogenous recombinant apelin-13 peptide was purchased from Sigma (St. Louis, MO). The makers of pericytes were PDGFR-B (ab69506, Abcam, US), NG2 (SC-20162, Santa Cruz, CA), and Desmin (ab6322, Abcam, US). Antiapelin and anti-APJ were purchased from Abcam company (ab59469, ab125213, and ab84296, Abcam, US), respectively. CellTiter 96 AQueous One Solution was used in cell Proliferation Assay (Promega, US). Lentiviral vector knockout of apelin was constructed and purchased from GeneChem Co., Ltd. (Shanghai, China). Bcl-2 and Bax antibody were obtained from Cell Signaling Technology (\#2870; \#2772, CST, US).

2.2. Primary Rat Pericyte Cells Isolation, Culture, and Treatment. All experiments were performed in accordance with the Research Ethics Committees of the People's Hospital, Peking University, China. We isolated primary rat retinal pericyte cells from the retinal microvessel of Sprague-Dawley (SD) rats, using a modified method of previously published articles [27-29]. Briefly, eyes from SD rats (4-6 weeks, 150-200 g) were incubated with cold Dulbecco's phosphatebuffered saline (PBS) containing penicillin-streptomycin antibiotic $(500 \mathrm{U} / \mathrm{mL})$ for $10 \mathrm{~min}$. The retinas were removed and cut into $1 \times 1 \mathrm{~mm}$ small pieces and then incubated with collagenase I (Roche Applied Science, Mannheim, Germany) for $30-45 \mathrm{~min}$ at $37^{\circ} \mathrm{C}$. The digested retina were filtered through $70 \mu \mathrm{m}$ and $40 \mu \mathrm{m}$ nylon mesh (Falcon, $\mathrm{BD}, \mathrm{US})$ and then centrifuged. Rat retinal pericytes were purified with Dynabeads Pan Mouse IgG (Invitrogen Dynal AS, Norway) according to the instructions. Before use, we washed the Dynabeads $(25 \mu \mathrm{L})$ in Dulbecco's Modified Eagle's Media (DMEM) (Hyclone, US), and we added $1 \mu \mathrm{L}$ mouse anti-desmin monoclonal antibody (ab6322, abcam, US) and then incubated them overnight at $4^{\circ} \mathrm{C}$. The cell pellets were suspended in DMEM containing 10\% fetal bovine serum (Gibco, US) and incubated with Dynabeads conjugated mouse anti-desmin monoclonal antibody for $30 \mathrm{~min}$ at $37^{\circ} \mathrm{C}$, with gentle rotation. After washing, the beadbound pericytes in pericyte medium (Sciencell Inc., US) were suspended at $37^{\circ} \mathrm{C}$ in a humidified atmosphere of a $5 \% \mathrm{CO}_{2}$ incubator. Pericytes between passages three and five were used throughout the study.

In chemical hypoxia-induced pericytes injury models, $150 \mu \mathrm{mol} \mathrm{CoCl}{ }_{2}$ was used according to the previous report [21]. In the viability assay, we treated pericytes with different concentrations of apelin $(10,100$, and $1000 \mathrm{ng} / \mathrm{mL})$ and knockdown of apelin was performed via lentivirus vector. We incubated control group cells in pericyte medium.

2.3. Immunofluorescent Staining. Immunofluorescent staining of membranes tissue was described in our previous published study [20,30]. Briefly, 12 fibrovascular membranes with proliferative diabetic retinopathy were surgically obtained during vitrectomy. In a similar manner, 10 macular preretinal membranes were obtained and served as control. Immunofluorescence staining was performed on the frozen sections of the fibrovascular membranes and of the control membranes by staining with rabbit anti-apelin (ab59469, 1:100, Abcam, US) or rabbit anti-APJ (ab84296, 1:200, Abcam, US). The human patients study protocol was approved by the Ethical Committee and Institutional Review Board of Peking University People's Hospital (Beijing, China) and was conducted in accordance with the Declaration of Helsinki. Written informed consent was obtained from each study subject.

Pericytes which were cultured on cover slides (Fisher, US) were fixed with $4 \%$ paraformaldehyde and incubated in $0.3 \% \mathrm{H}_{2} \mathrm{O}_{2}$ and $0.1 \%$ triton $\mathrm{X}-100$ to quench endogenous peroxidase activity and penetrate the cytomembrane. Then, the cells were incubated in 3\% blocking goat serum for $1 \mathrm{~h}$ and then incubated with anti-PDGFR- $\beta$ (ab69506, 1:100, Abcam, US), NG2 (SC-20162, 1: 100, Santa Cruz, CA), desmin (ab6322, 1:200, Abcam, US), apelin (1:100, Abcam, US), and APJ $\left(1: 200\right.$, Abcam, US) overnight at $4^{\circ} \mathrm{C}$. The following day, pericytes were incubated with the relevant fluorescenceconjugated secondary antibody (1:200, Invitrogen, US) for $2 \mathrm{~h}$ at room temperature. Images were obtained with Nikon $50 \mathrm{i}$ fluorescent microscope (Nikon, Tokyo, Japan) under 200x magnifications.

2.4. Lentivirus-Mediated shRNA Knockdown of Apelin Expression and Transfection. The knockdown of Apelin (Rattus, NM_031612.2, GI:52345441) was induced by a lentivirusmediated RNA interference vector (GeneChem Co., Ltd., Shanghai, China). The small interfering RNA (siRNA) target sequences were selected: \#1, $5^{\prime}$-GAGGAGAGATAGAAACAGA-3'; \#2, $5^{\prime}$-GGAGGATGTTGGCTGAGAA-3'; \#3, $5^{\prime}$-GTTTGCCTTTCTTGACAAA-3'; and \#4, 5'-CAGATGAGTTCTCTTCTCT-3' . The lentivirus-GFP (LV-GFP) which included the GFP gene and did not include the apelin interference sequence served as negative control. For lentivirus transduction, pericytes were cultured at $5 \times 10^{4}$ cells/well into 6 -well culture plates. After being grown to $70 \%$ confluence, cells were transduced with shRNA lentivirus at 
a multiplicity of infection (MOI) of 100. Cells were harvested at 24 hours after infection, and transfection efficiency was evaluated by immunofluorescence staining. The knockdown efficiency of apelin was evaluated by RT-PCR and western blot analysis.

2.5. Cell Viability/Cell Proliferation. Pericyte viability was measured by MTS assay, according to the manufacturer's instructions (CellTiter 96 AQueous One Solution Assay; Promega, Madison, WI, US). 5000 cells/well were seeded into a 96-well plate and incubated with different concentrations of apelin $(1,100$, and $1000 \mathrm{ng} / \mathrm{mL})$ for $24 \mathrm{~h}$. At the end of the incubation, $10 \mu \mathrm{L}$ MTS solution was added into each well and incubated for $1 \mathrm{~h}$. The absorbance wavelength was evaluated with microculture plate reader (Model 550; Bio-Rad, Tokyo, Japan) at $490 \mathrm{~nm}\left(\mathrm{OD}_{490}\right)$. Each experiment was performed in five wells and repeated at least three times.

2.6. Edu Assay. Pericytes proliferation was assessed using a Cell-Light EdU Apollo 643 in vitro Imaging Kit (RuiBo. Inc., Guangzhou, China). Briefly, $1 \times 10^{4}$ cells/well, which was pretreated with apelin, was plated in 96-well plates for $24 \mathrm{~h}$. Following the incubation interval, $10 \mu \mathrm{mol}$ 5-ethynyl2 -deoxyuridine (Edu) medium was added to each well and incubated for $2 \mathrm{~h}$. After washing twice with PBS, pericytes were fixed with $4 \%$ paraformaldehyde for $30 \mathrm{~min}$ and washed with $2 \mathrm{mg} / \mathrm{mL}$ glycine solution for $5 \mathrm{~min}$ in order to neutralize paraformaldehyde and assure a good staining system. The cells were incubated in $100 \mu \mathrm{L} 1 \mathrm{x}$ Apollo staining solution for $30 \mathrm{~min}$ and the nuclei were dyed with Hoechst 33342. The images were obtained under 10x magnification, using a Nikon 50i fluorescent microscope. Each experiment was performed in five wells and repeated at least three times.

2.7. Cell Migration/Transwell Assay. Transwell assay was used for evaluating cell migration assays. Briefly, $100 \mu \mathrm{L}$ of pericyte suspension $\left(1 \times 10^{5}\right.$ cells $\left./ \mathrm{mL}\right)$ was added to the upper chamber and $600 \mu \mathrm{L}$ medium containing apelin, hypoxia medium, or DMEM (control) to the lower chamber, respectively. The chambers were incubated for $6 \mathrm{~h}$ at $37^{\circ} \mathrm{C}$. The filters were fixed with $4 \%$ paraformaldehyde for $15 \mathrm{~min}$ and we subjected the nuclei to DAPI staining for $10 \mathrm{~min}$. The remaining cells on the upper surface of the filter were removed by wiping with a cotton swab. The number of migrated cells were quantified by counting in five random fields (10x magnification), using a Nikon 50i fluorescence microscope. The data are shown as the mean \pm standard deviation (SD). Each experiment was repeated at least three times.

2.8. TUNEL Assay. Pericyte apoptosis after hypoxia was evaluated by terminal deoxynucleotidyl transferase-mediated dUTP nick end-labeling (TUNEL) assay. TUNEL staining was performed on cell coverslips using a commercial kit (In Situ Cell Death Detection Kit; Roche Applied Science, USA), according to the manufacturer's recommended instructions. TUNEL-positive cell nuclei were visualized as green fluorescence and images performed under 10x magnification. Finally, the percentage of TUNEL-positive cells was calculated in five microscopic fields of each slide.
2.9. Quantitative Real-Time PCR. Total RNA was isolated from cultured pericytes using Trizol reagent (Invitrogen, CA, US), and we determined the concentration and integrity of total RNA with UV spectrophotometry (NANODROP 2000C, Thermo, US). We used the Fermentas reverse transcription system (Fermentas, St. Leon-Rot, Germany) to reverse RNA $(1 \mu \mathrm{g})$ into first strand CDNA, using a realtime PCR system (PikoReal 96 PCR system, Thermo Scientific). The PCR solution system contained $1 \mu \mathrm{L}$ of cDNA (1:20 diluted), specific primers $1 \mu \mathrm{L}$ (10 pmol), $3 \mu \mathrm{L}$ DEPCwater, and $5 \mu \mathrm{L}$ of SYBR Select Master Mix (Invitrogen), with a final volume of $10 \mu \mathrm{L}$. Each sample was measured in triplicate wells. Primers used were as follows: $\beta$-actin Forward: $5^{\prime}$-TGGCTCTATCCTGGCCTCACT-3', $\beta$-actin Reverse: $5^{\prime}$-GCTCAGTAACAGTCCGCCTAGAA-3'; rat apelin Forward: $5^{\prime}$-GATGGAGAAAGGCGAAGAAAG-3', rat apelin Reverse: $5^{\prime}$-GGTGAGAGATGAGACCACTTGT$3^{\prime}$. The standard PCR conditions included $2 \mathrm{~min}$ at $50^{\circ} \mathrm{C}$ and $10 \mathrm{~min}$ at $95^{\circ} \mathrm{C}$, followed by 35 cycles of extension at $95^{\circ} \mathrm{C}$ for $15 \mathrm{~s}, 60^{\circ} \mathrm{C}$ for $30 \mathrm{~s}$, and $72^{\circ} \mathrm{C}$ for $30 \mathrm{~s}$. The mRNA expression was normalized to the expression level of ACTB. We calculated the changes in mRNA expression according to the $2^{-\Delta \Delta} \mathrm{CT}$ method, with $\Delta \mathrm{CT}=\mathrm{C}_{\text {Target gene }}-\mathrm{CT}_{\mathrm{ACTB}}$ and $\Delta \Delta \mathrm{CT}=\Delta \mathrm{C}_{\text {Treatment }}-\Delta \mathrm{CT}_{\text {Control }}$. Each experiment was repeated at least three times.

2.10. Western Blot Analysis. Pericytes were harvested and lysed in RIPA buffer (1\% Nonidet P-40, 0.5\% sodium deoxycholate, $0.1 \%$ SDS in PBS) and centrifuged at 15,000 rpm for $15 \mathrm{~min}$ at $4^{\circ} \mathrm{C}$. The membranes were blocked with $5 \%$ nonfat milk for $1 \mathrm{~h}$ and then incubated overnight at $4^{\circ} \mathrm{C}$ with primary antibody: rabbit anti-Bcl-2, (\#2870; 1:1000, CST); rabbit anti-Bax, (\#2772; 1:1000; CST); rabbit anti-Apelin (ab125213; 1:500; abcam); and rabbit anti-APJ (ab84296; $1: 1000$, abcam). The membranes were incubated with goat anti-rabbit horse-radish peroxidase- (HRP-) conjugated secondary antibody $(1: 3000$, DAKO, Japan) for $1 \mathrm{~h}$ at room temperature. The density of each band was analyzed with Image J software. Each experiment was repeated at least three times.

2.11. Statistical Analysis. The results were expressed as mean \pm SD. Difference between two groups was compared with an independent sample $t$-test (SPSS17.0 software, Chicago, IL). Two-tailed $P<0.05$ was considered to indicate statistical significance. Differences among groups were assessed using one-way analysis of variance (ANOVA), followed by Dunnett's test. A value of $P<0.05$ was considered as significantly different. We repeated all experiments at least three times, and representative experiments are shown.

\section{Results}

3.1. Immunohistochemical Expression of APJ in Fibrovascular Membranes. Expression of APJ was detected in the specimens of all fibrovascular membranes of the proliferative diabetic retinopathy (PDR) group with strong staining for APJ (Figure 1). Colocalization of pericyte markers desmin and APJ were observed in all specimens of the PDR group 
APJ

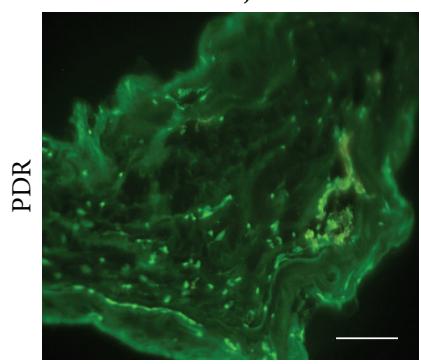

(a)

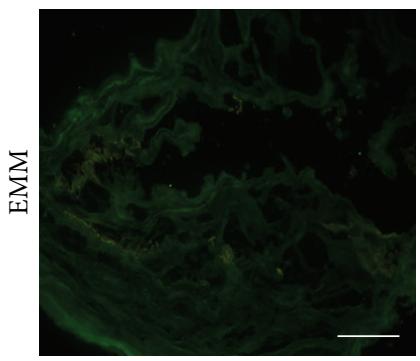

(e)

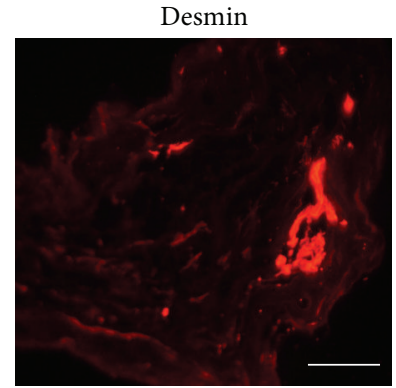

(b)

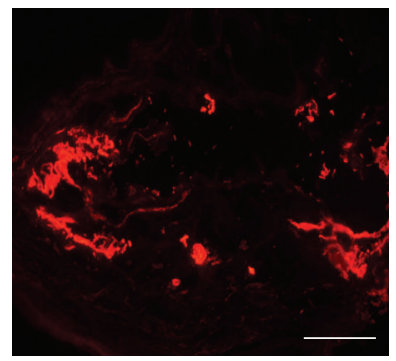

(f)

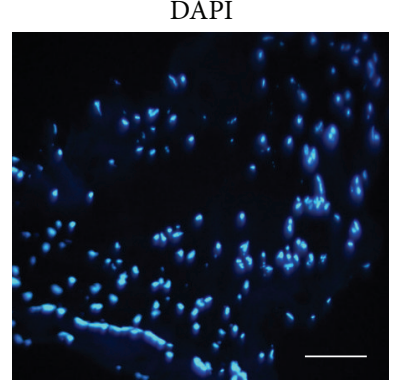

(c)

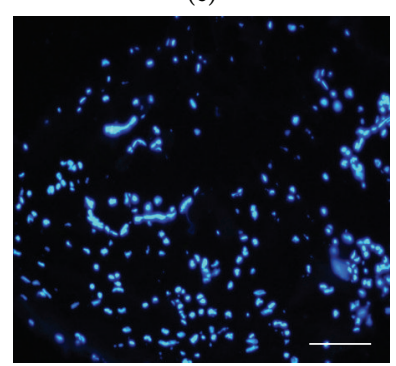

(g)

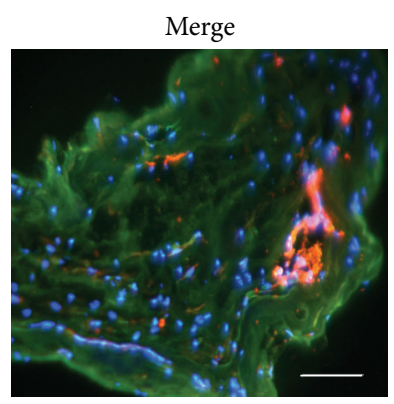

(d)

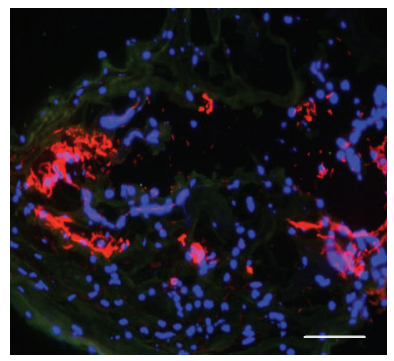

(h)

FIGURE 1: Immunostaining of APJ and pericyte in fibrovascular membranes. (a-d) Immunostaining for APJ (a), pericyte marker desmin (b), and DAPI (c) in fibrovascular membranes from eyes with proliferative diabetic retinopathy. Staining intensities of APJ were strong and were colocalized with pericyte, as identified by desmin. (e-h) Staining of APJ (e), desmin (f), and DAPI (g) in epiretinal macular membranes $(E M M)$ of control patients without diabetic retinopathy. None of APJ (e) and staining of desmin (f) were observed. Scale bar $=100 \mu \mathrm{m}$.

$(n=12)$ (Figures $1(\mathrm{a})-1(\mathrm{~d}))$. None of the membranes removed from the eyes of the epithelial macular membrane (EMM) group showed specific staining of APJ $(n=10)$ (Figures 1(e)-1(h)). Our previous study also demonstrated that vitreous concentrations of apelin were significantly higher in the PDR group than in the EMM group [30].

\subsection{Cultivation and Identification of Primary Rat Retinal Peri-} cytes. Primary rat retinal pericytes were isolated by Magnetic Dynabeads and formed cell clusters floating in cell medium (Figure 2(A)). At day 7, the primary pericytes got adherence, and the colony formed and grew (Figure 2(B)). Generally, primary pericytes confluenced at about day 14 . When pericytes were passaged, the growth and adherence became rapid obviously, which got adherence about 4-6 hours and passaged about 4-5 days. Primary rat retinal pericytes showed irregular triangular cell bodies, with thick filaments in the cytoplasm and a plump nucleus (Figure 2(C)). As specific markers for pericyte, desmin, PDGFR- $\beta$, and NG2 were used to confirm the purity of cultured primary rat pericyte cells, which was approximately 95\% (Figure 3(b)).

3.3. Expression of Apelin and APJ Receptor in Pericytes. Prior to exploring the effects of apelin in rat retinal pericytes, we carried out immunofluorescence staining to detect the expression of apelin/APJ in pericytes. We observed low apelin immunoreactivity in normal pericyte culture, which showed weak and diffuse expression in the cytoplasm (Figure 3(a)(A), (B)). In similar way, the APJ staining was moderate, which was expressed in cytoplasm membrane (Figure 3(b)(A), (B)). However, after exposure under hypoxia for $12 \mathrm{~h}$, the expression of apelin represented obvious stronger cytoplasm staining (Figure 3(a)-(C), (D)), accompanied by expanding stronger APJ immunoreactivities in cytoplasm and cytoplasm membrane (Figure 3(b)-(C), (D)). In addition, the results of western blot about apelin and APJ under hypoxia support the change of immunofluorescence staining (Figures 3(c) and $3(\mathrm{~d}))$. The expression of apelin and APJ under hypoxia was upregulated 2.5-fold and 1.9-fold, respectively $(P<0.05)$.

3.4. Detection of Interference Efficacy of Lentivirus-Apelin. Quantitative Real-Time PCR in NRK-52E and IEC6 cells demonstrated that LV-Apelin (\#4) was the most efficient shRNA, in which the RNA level of apelin was decreased by more than $70 \%$ (data was not shown). Then, we tested the knockdown efficiencies of LV-Apelin in pericytes. When MOI is 100 , the result of immunofluorescence staining showed that interference efficacy achieved 90\% (Figure 4(a)). After that, the qRT-PCR in pericyte revealed the apelin level was decreased by $75 \%$ in $\mathrm{LV}$-apelin group compared to $\mathrm{LV}$ GFP control group $(P<0.01)$ (Figure $4(b))$. In a similar way, western blot demonstrated that the apelin level was downregulated by $64 \%(P<0.05)$ (Figures $4(\mathrm{c})$ and $4(\mathrm{~d})$ ).

\subsection{Apelin-Stimulated Cell Proliferation and Migration in} Normoxia. Experiments were performed to evaluate whether apelin had any effect on pericytes proliferation and migration in normoxia. Pericytes were incubated with apelin at different concentrations $(1,10,100$, and $1000 \mathrm{ng} / \mathrm{mL})$ for $24 \mathrm{~h}$. Among the various concentrations, MTS assay results show that $100 \mathrm{ng} / \mathrm{mL}$ group significantly increased pericytes viability, compared with the control groups (Figure 5(B)) $(100 \mathrm{ng} / \mathrm{mL}$ 


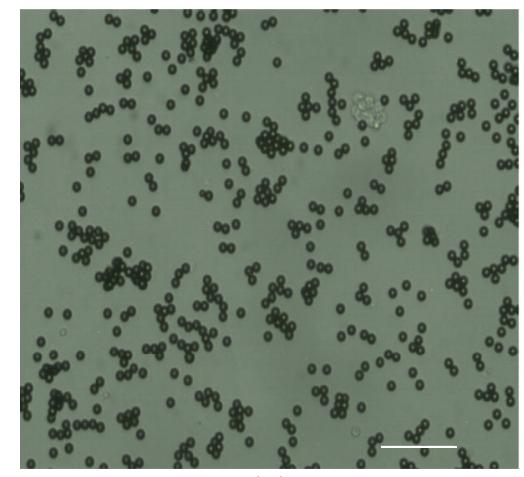

(A)
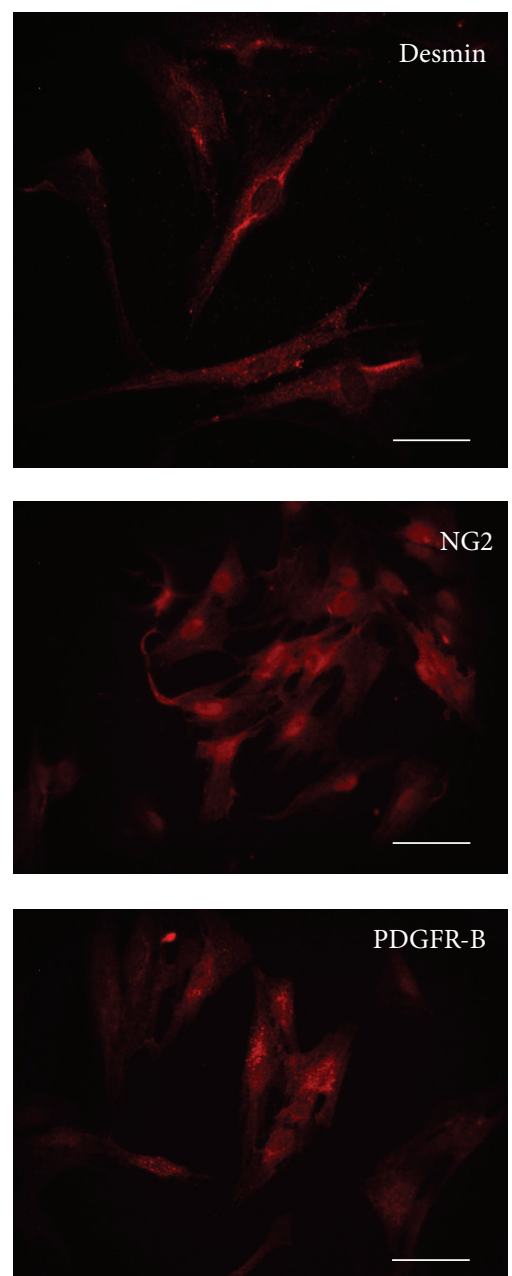

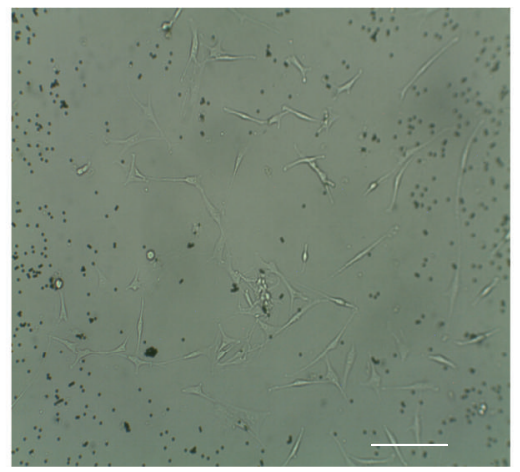

(B)

(a)
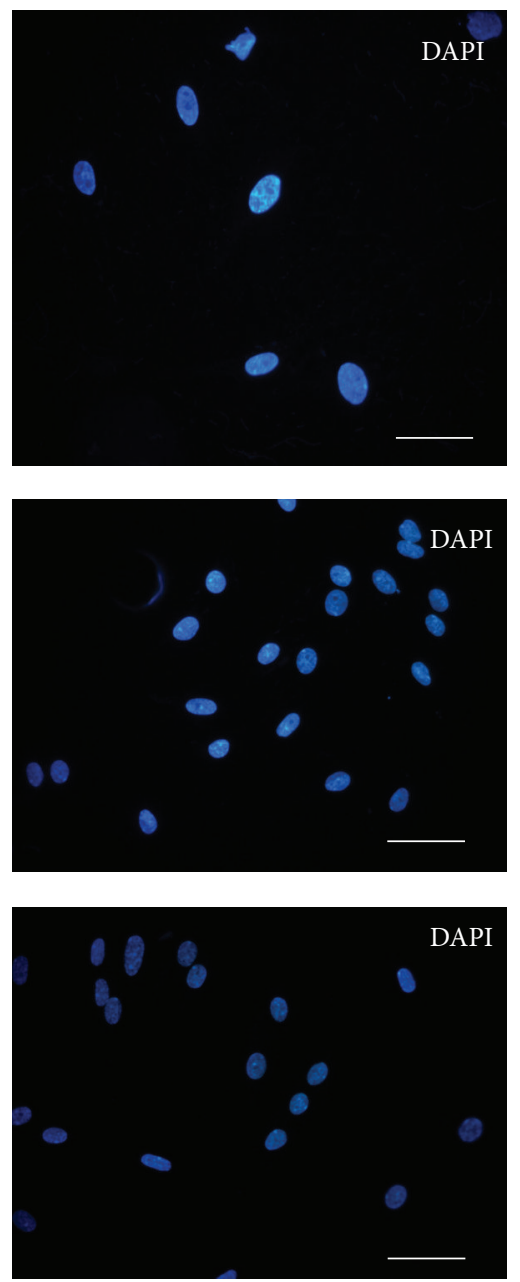

(b)

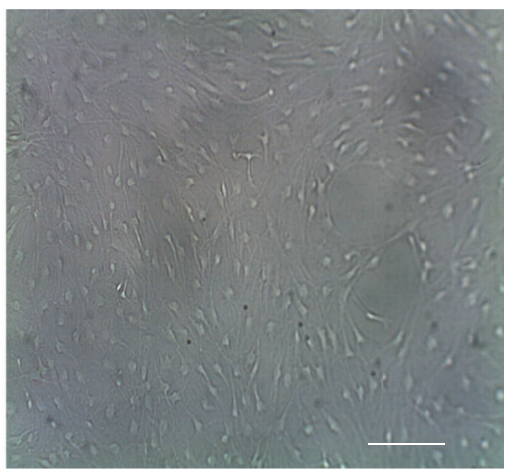

(C)
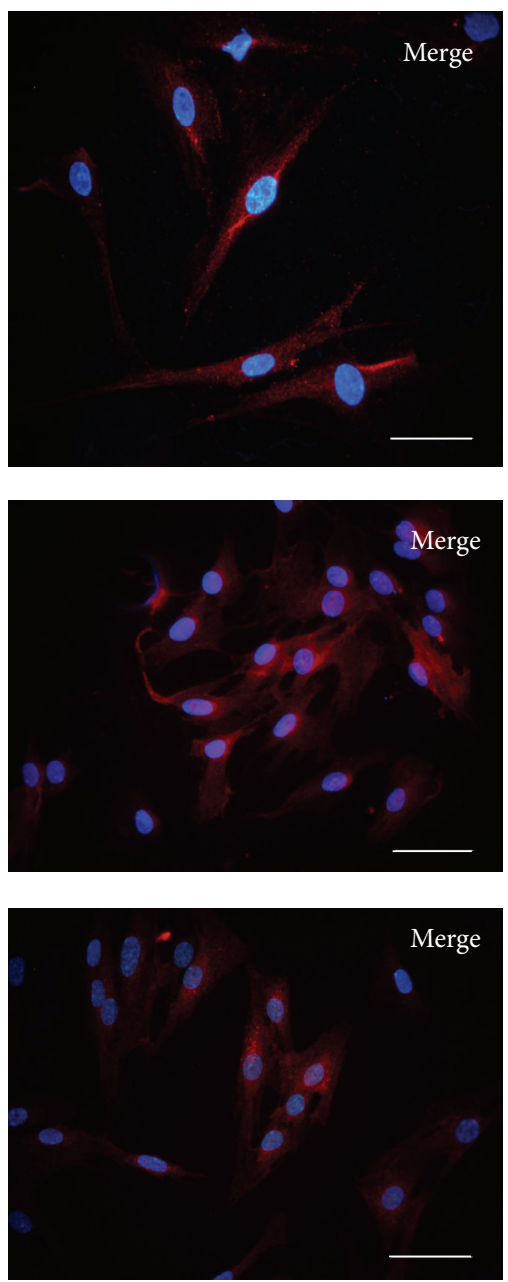

FIGURE 2: Morphology and immunofluorescent staining were identified in primary rat retinal pericytes. (a) Microscopic image of retinal pericytes, showing cell mass isolated by Dynabeads (A), at 7 days, pericytes got adherence and formed cells cluster (B), at passage 1 noncontact-inhibited growth of pericyte and irregular triangular cell bodies, with thick filaments in the cytoplasm and a plump nucleus (C). Scale bar $=200 \mu \mathrm{m}$. (b) Immunofluorescence staining with anti-desmin, NG2, and PDGFR-B antibody for primary rat retinal pericytes, respectively. Scale bar $=100 \mu \mathrm{m}$. 


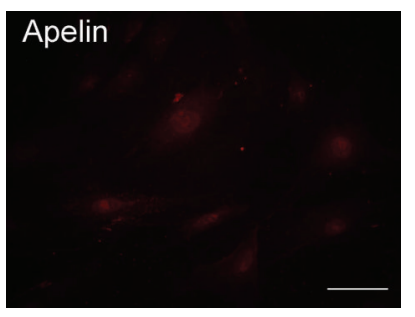

(A)

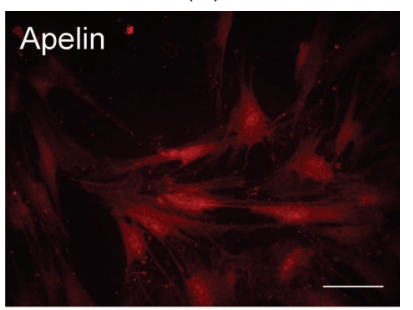

(C)

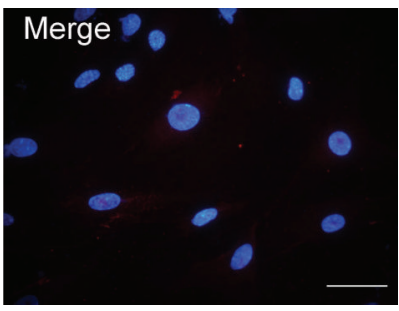

(B)

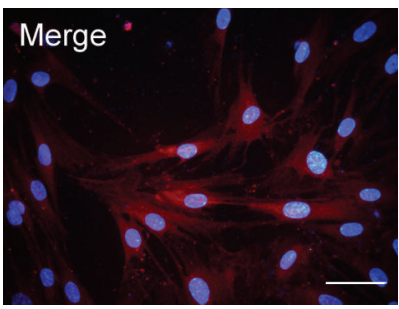

(D)

(a)
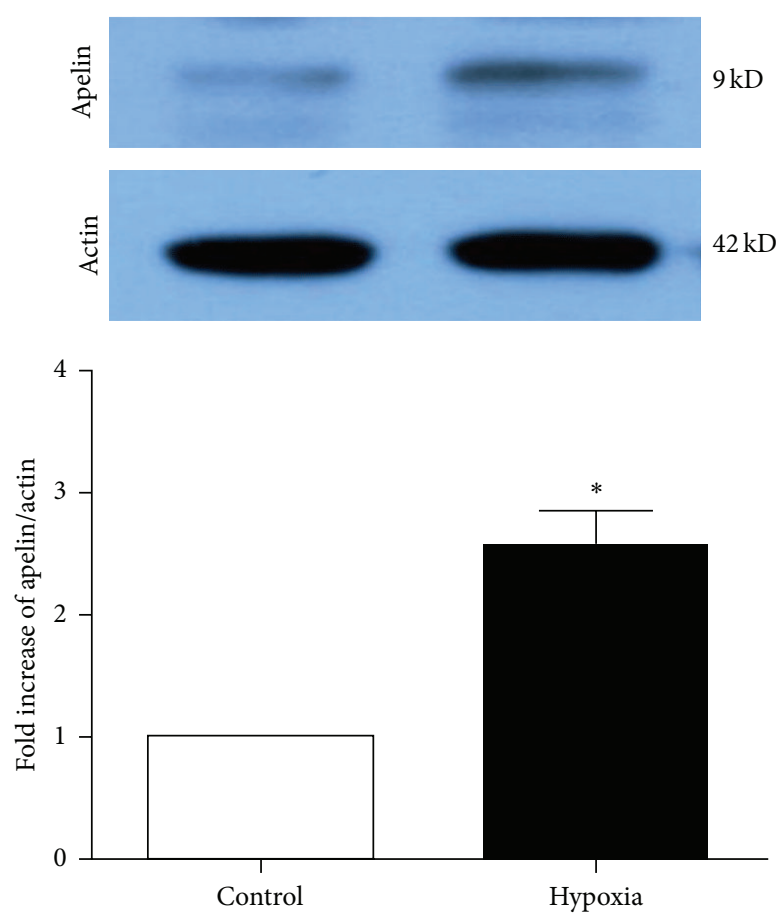

(c)

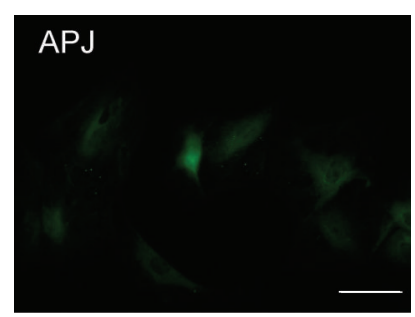

(A)

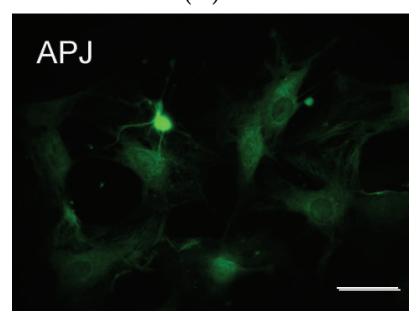

(C)

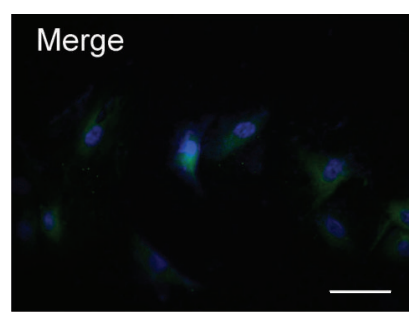

(B)

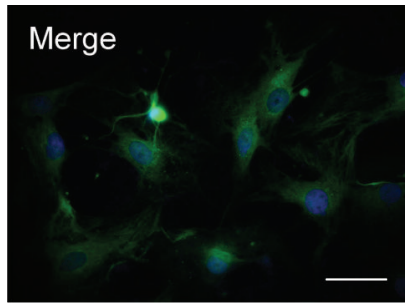

(D)

(b)
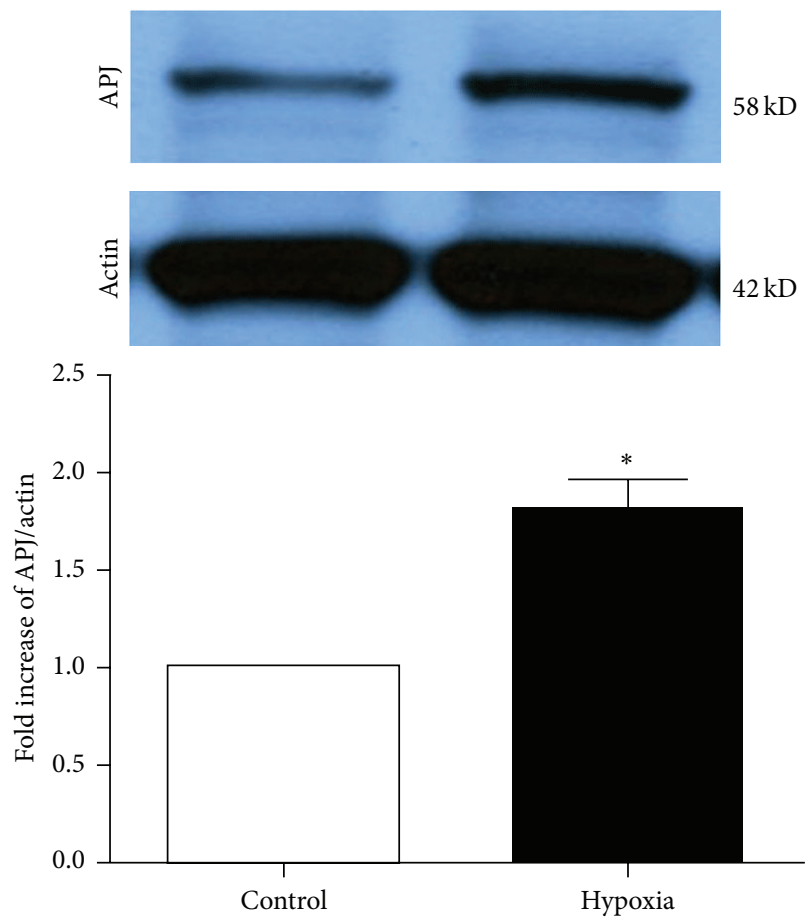

(d)

FIGURE 3: The cellular localization of apelin/APJ. (a) Apelin immunoreactivity was found weak and diffused in the cytoplasm of normal (A) but showed more intense cytoplasmic staining in hypoxic pericyte (C). Scale bar $=100 \mu \mathrm{m}$. Similarly, (b) compared with restricted cytomembrane expression in normal (A) and hypoxia (C) pericyte, APJ localization expanded and brightened in the cytoplasm and cytoplasm membrane (apelin in red, APJ in green, and DAPI in blue). Scale bar $=100 \mu \mathrm{m}$. (c) and (d) Western blot analysis shows that the expression of apelin and APJ under hypoxia was upregulated 2.5-fold and 1.9-fold, respectively $(P<0.05)$.

versus control, ${ }^{* *} \mathrm{P}<0.01 ; 1000 \mathrm{ng} / \mathrm{mL}$ versus control, ${ }^{*} \mathrm{P}<$ $0.05)$.

Edu experiment was used to detect pericyte proliferation at different concentrations of apelin $(10 \mathrm{ng} / \mathrm{mL}$ and $100 \mathrm{ng} / \mathrm{mL}$ ). The number of proliferative cells was significantly higher in the apelin-treated group, compared with the control group (Figure 5(A)).

In the cell migration assay, cells were measured in a modified Boyden Chamber in which pericytes migrated through a porous membrane. The mean number of migrated pericytes incubated with apelin $(1-100 \mathrm{ng} / \mathrm{mL})$ was significantly higher than the mean number of the control group $(P<0.05)$ (Figures 5(C) and 5(D)).

3.6. The Effects of Apelin and Lentivirus Knockdown Apelin for Cell Proliferation and Migration in Hypoxia. Furthermore, we carried out experiment to study the effect of apelin for pericyte under hypoxia. We found that the viability of 


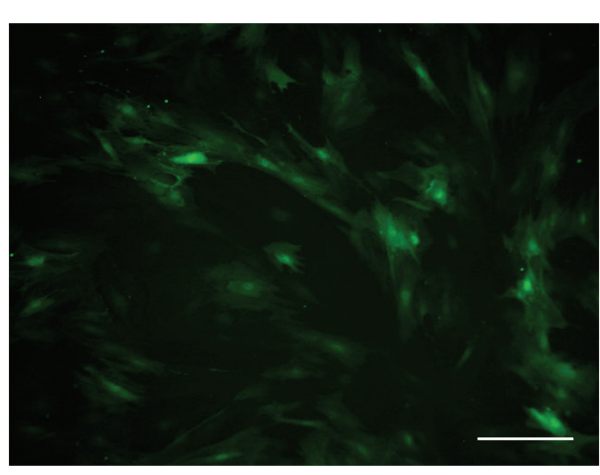

(a)

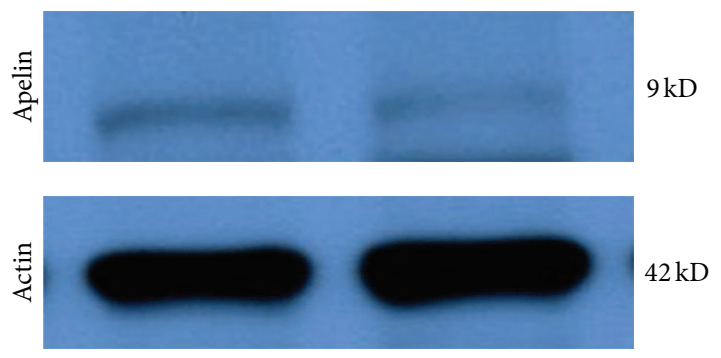

(c)

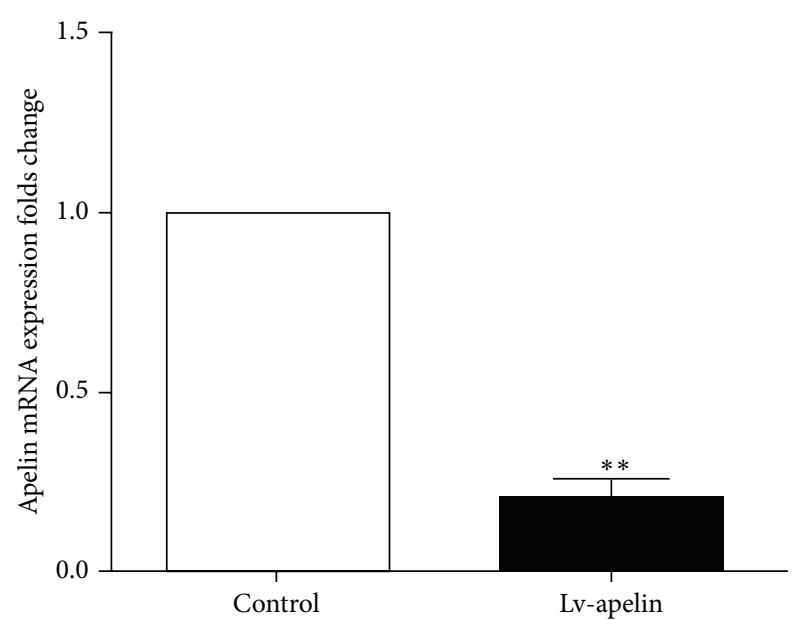

(b)

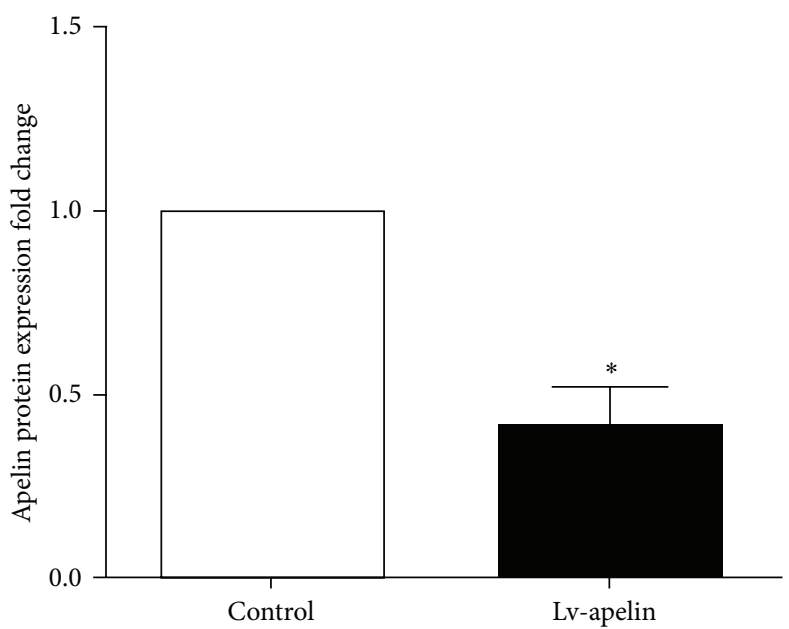

(d)

Figure 4: Pericytes transducted with Lentivirus-Apelin. (a) Immunofluorescence staining of LV-apelin infection in pericyte. Interference efficacy arrived about $90 \%$, MOI $=100$. Scale bar $=100 \mu \mathrm{m}$. (b) The mRNA expression of LV-Apelin was decreased by $75 \%$ after blocking by siRNA sequence $(P<0.01)$. (c and $d)$. The western blot analysis shows that protein of expression of LV-Apelin decreased by $64 \%(P<0.05)$.

pericytes incubated with $\mathrm{CoCl}_{2}$ was decreased obviously time dependently. The viability of pericytes was reduced by $27 \%$ at $6 \mathrm{~h}$ and by $40 \%$ at $12 \mathrm{~h}$ after stimulation by $150 \mu \mathrm{mol} / \mathrm{L} \mathrm{CoCl}_{2}$, respectively. However, the viability of pericytes stimulated by apelin was significantly enhanced, compared with the $\mathrm{CoCl}_{2}$ group $\left(8 \mathrm{~h}\right.$ versus control ${ }^{*} P<0.05 ; 12 \mathrm{~h}$ versus control ${ }^{* *} P<0.01$ ) (Figure 6(A)).

Our study showed that under hypoxia the viability of cells treated with apelin was significantly increased. Meanwhile, the viability of cells in the LV-apelin knockout group was significantly reduced $\left(\mathrm{CoCl}_{2}\right.$ versus apelin, $P<0.05$; LV-GFP versus LV-apelin, $P<0.05 ; \mathrm{CoCl}_{2}$ versus LVapelin, $P<0.01$ ), which suggests that apelin can stimulate pericyte viability (Figure 6(B)). We also detected migration of pericytes under hypoxia. Compared with the control group, the mean number of migrated pericytes under hypoxia and combination with LV-apelin knockdown decreased significantly. However, in the group treated with apelin, the mean number of migrated pericytes was increased under hypoxia (Figures 6(C) and 6(D)).

\subsection{Apelin Protected Pericytes against Apoptosis Induced by} Hypoxia via Bcl-2/Bax Restoration and Caspase 3 Pathway. In the cell viability experiment, hypoxia resulted in a $27 \%$ decrease in pericyte viability. However, cell viability increased significantly in pericytes pretreated with $100 \mathrm{ng} / \mathrm{mL}$ of apelin for $12 \mathrm{~h}$. To further evaluate the effects of apelin on cell death, we used TUNEL staining to detect DNA fragmentation and cell death in hypoxia-treated pericytes with and without apelin treatment. We pretreated pericytes with apelin for $12 \mathrm{~h}$ and then exposed these pericytes to hypoxia for $12 \mathrm{~h}$. In the percentage of apoptotic cells showing green, approximately $30 \%$ of cell death was blocked by the apelin treatment (Figure 7).

Active-caspase 3 protein is one of the key executioners of apoptosis. As shown in Figure 8, active-caspase 3 protein 

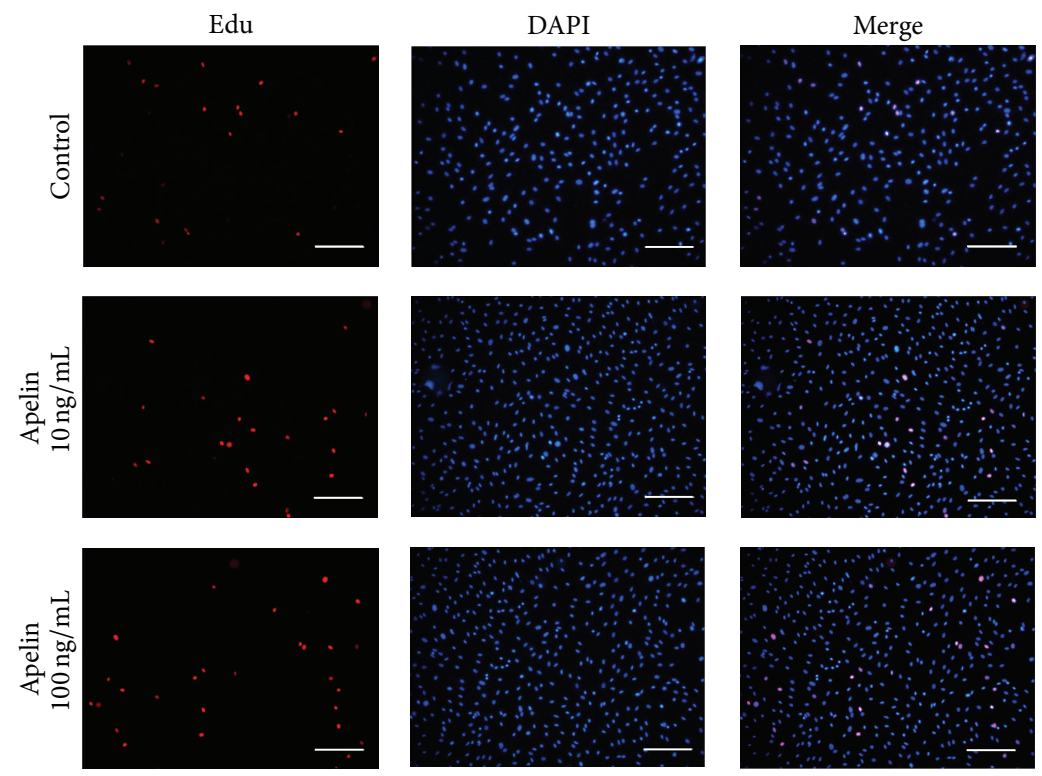

(A)

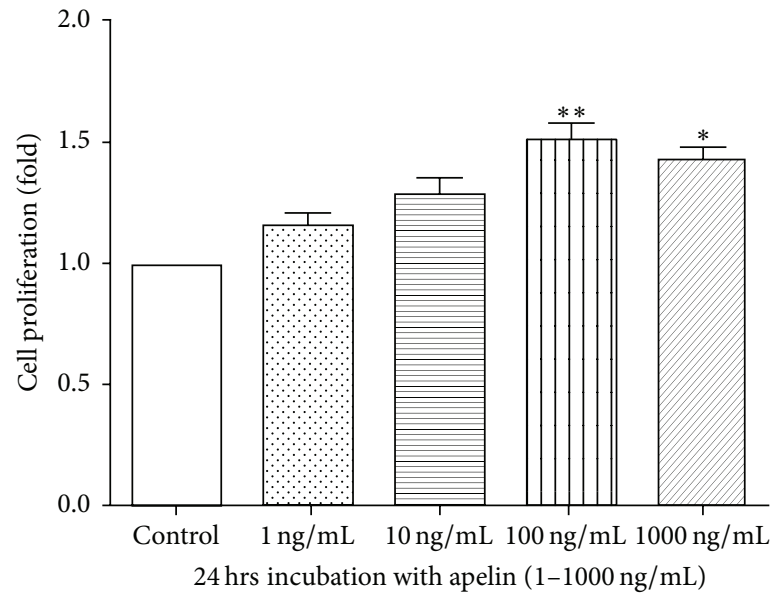

(B)

$24 \mathrm{hrs}$ incubation with apelin $(1-1000 \mathrm{ng} / \mathrm{mL})$
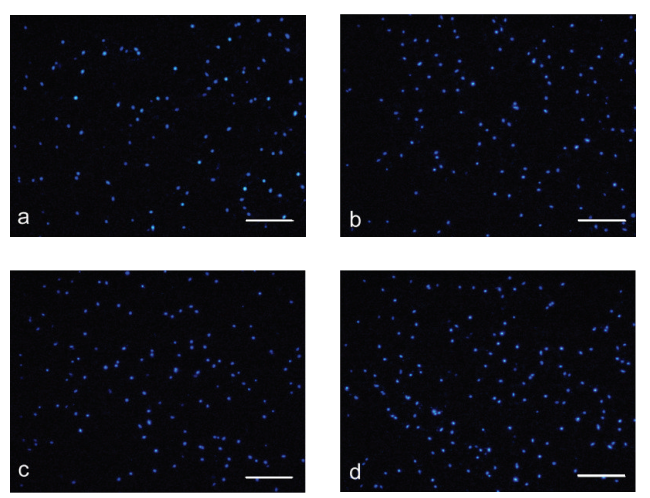

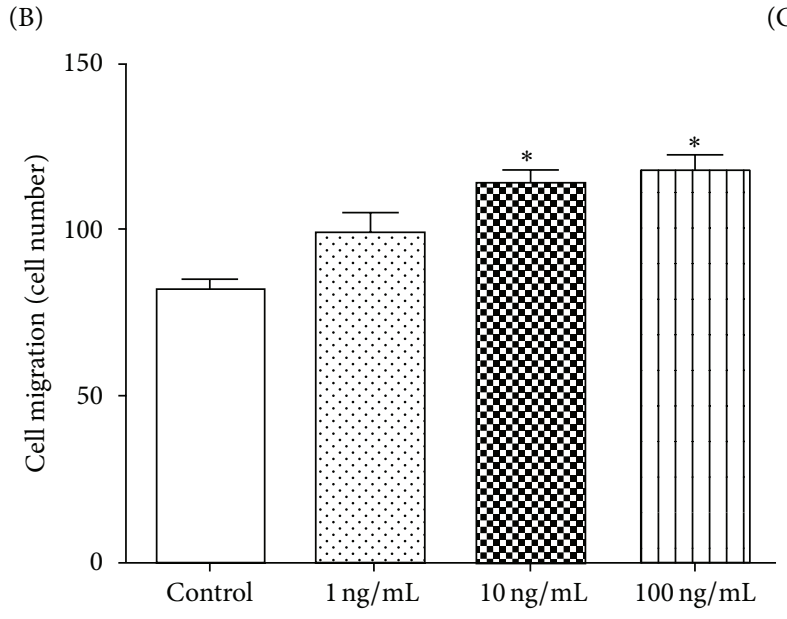

(D)

FIGURE 5: Effect of apelin on cell viability and migration under normoxia. (A) The Edu proliferation assay. Apollo staining (red) represents proliferating cells, and DAPI (blue) staining nuclei. Compared with the control group, the number of proliferating cells treated with apelin (10 or $100 \mathrm{ng} / \mathrm{mL}$ ) increased significantly. Scale bar $=200 \mu \mathrm{m}$. (B) The folds of apelin-treated cell viability compared with the control group $\left({ }^{*} P<0.05,{ }^{* *} P<0.01\right.$ versus untreated control); (C) and (D) pericyte migration in response to apelin treatment was measured using the transwell assay (a: control; b: $1 \mathrm{ng} / \mathrm{mL} ; \mathrm{c}: 10 \mathrm{ng} / \mathrm{mL}$; d: $100 \mathrm{ng} / \mathrm{mL},{ }^{*} \mathrm{P}<0.05$ versus untreated control). The data are expressed as means \pm standard deviation (SD). Scale bar $=200 \mu \mathrm{m}$. 


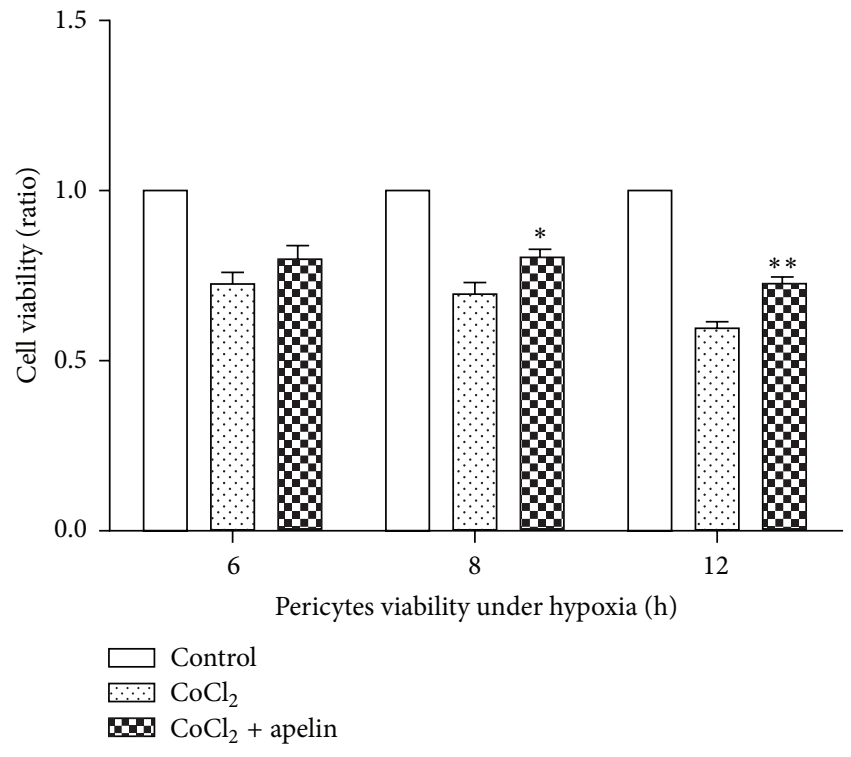

(A)
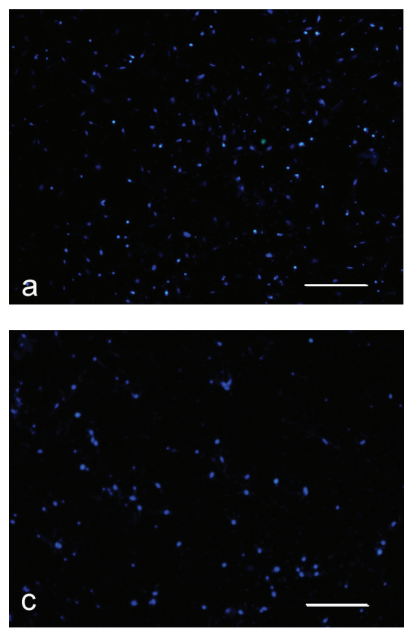
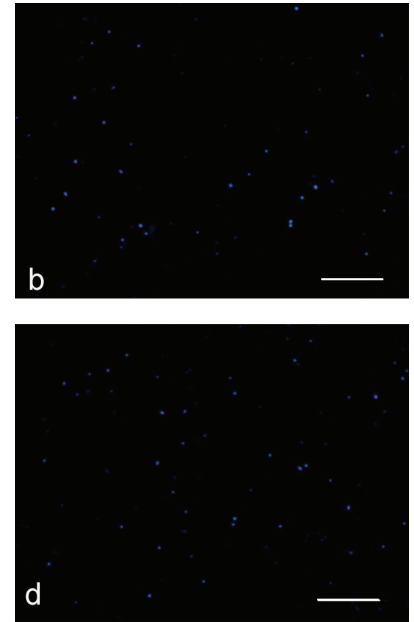

(C)

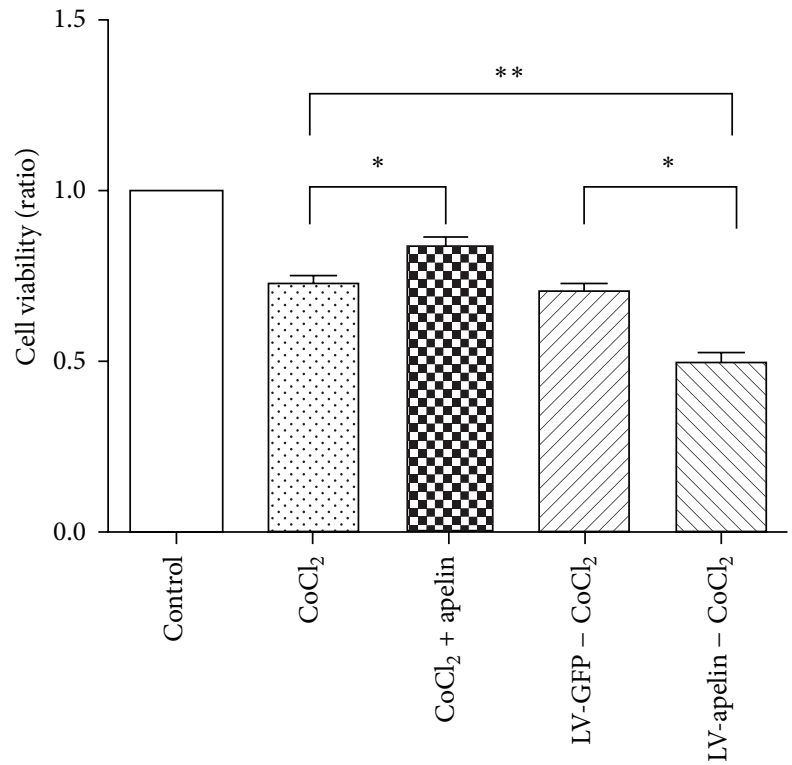

(B)

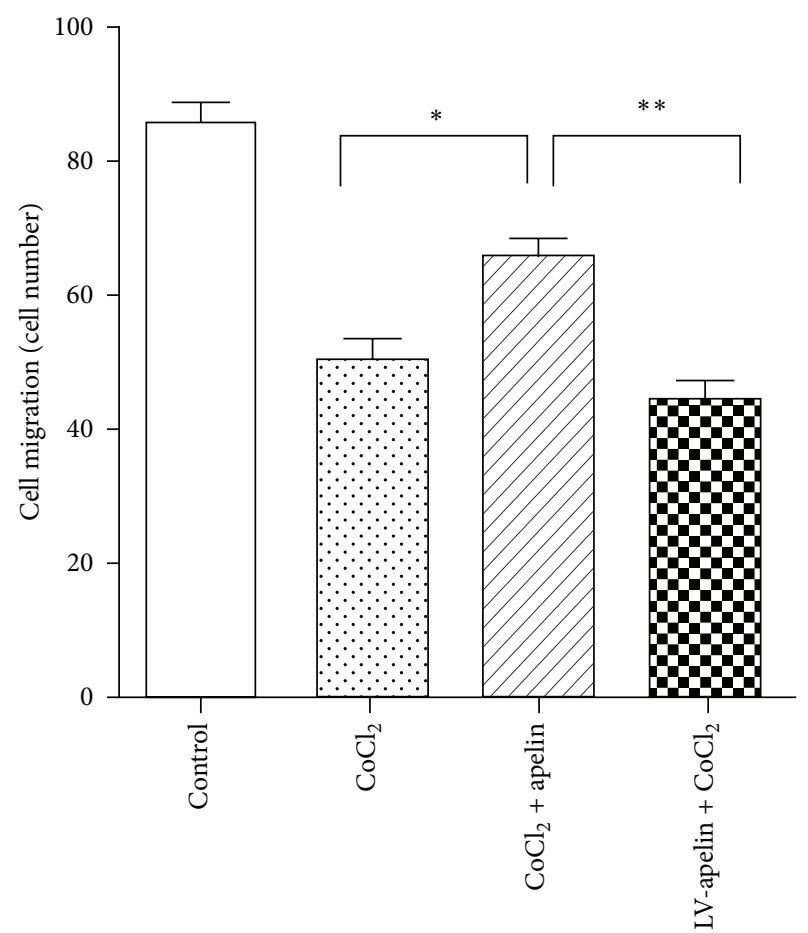

(D)

FIGURE 6: Effect of apelin on cell viability and migration under hypoxia. Cell viability was evaluated by MTS assay and migration was assessed with a transwell cell chamber. (A) In hypoxic pericytes, the viability of cells stimulated by apelin was significantly enhanced during $12 \mathrm{~h}$ (8 $\mathrm{h}$ versus $\mathrm{CoCl}_{2}{ }^{*} \mathrm{P}<0.05 ; 12 \mathrm{~h}$ versus $\left.\mathrm{CoCl}_{2}{ }^{* *} \mathrm{P}<0.01\right)$. (B) Viability of pericytes treated with apelin and LV-apelin knockout under hypoxia. Compared with the $\mathrm{CoCl}_{2}$ group, viability was significantly increased in the apelin group $(P<0.05)$. Moreover, cell viability was significantly reduced in the LV-apelin knockout group (LV-GFP versus $\mathrm{LV}$-apelin, $P<0.05 ; \mathrm{CoCl}_{2}$ versus $\mathrm{LV}$-apelin, $P<0.05$ ). (C) and (D) Pericyte migration induced by apelin under hypoxia (a: control; b: $\mathrm{CoCl}_{2} 150 \mu \mathrm{mol}$; and c: $\mathrm{CoCl}_{2} 150 \mu \mathrm{mol}+$ apelin $100 \mathrm{ng} / \mathrm{mL}$ ). The number of migrated cells per HPF is shown. Apelin versus $\mathrm{CoCl}_{2}{ }^{*} P<0.05$. Scale bar $=200 \mu \mathrm{m}$. 
TUNEL
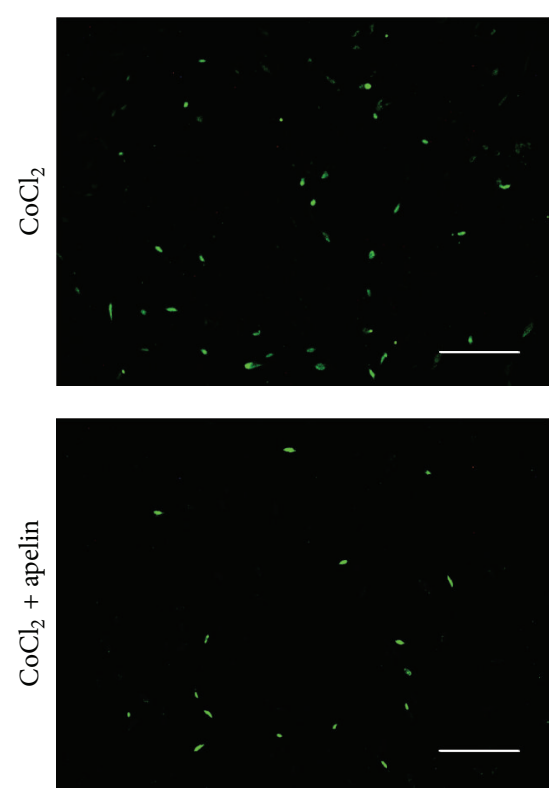

DAPI
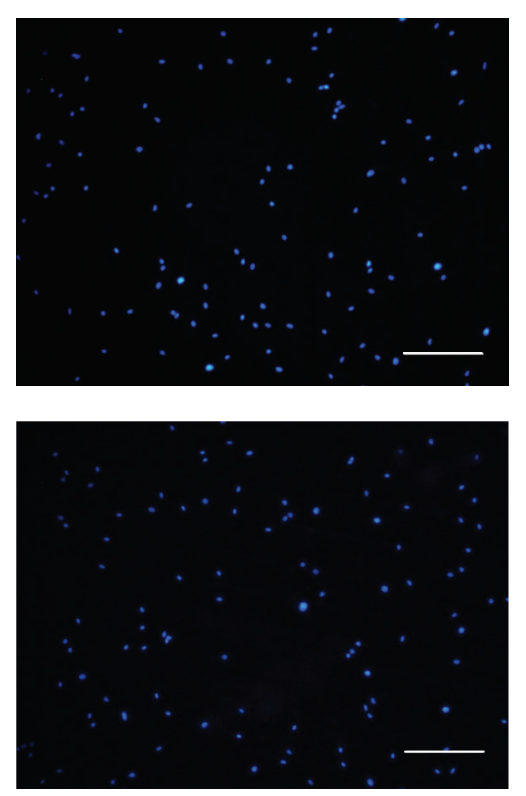

Merge
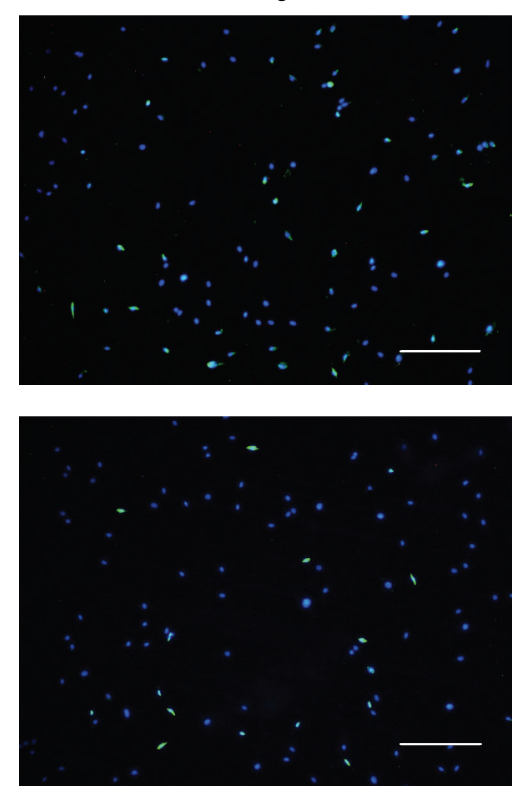

FIGURE 7: TUNEL staining was used to evaluate hypoxia-induced cell death. Cells were exposed to $100 \mathrm{ng} / \mathrm{mL}$ apelin for $12 \mathrm{~h}$ and then exposed to hypoxia for $12 \mathrm{~h}$. Apoptotic nuclei were visualized by TdT-mediated dUTP nick end-labeling (TUNEL). Scale bar $=200 \mu \mathrm{m}$.

level significantly increased $12 \mathrm{~h}$ after hypoxia injury $(P<$ 0.001, compared with the control group). Administration of apelin significantly decreased its levels after hypoxia injury $(P<0.01$, compared with hypoxia group). The expression of active-caspase 3 in hypoxia combination with LV-Apelin knockdown was similar to hypoxia group and its levels significantly increased $(P<0.01$, compared with control group) (Figures $8(\mathrm{a})$ and $8(\mathrm{~b}))$.

Likewise, we also detect bcl-2 and Bax expression in pericyte. Western blot analysis showed that apelin dosedependently induced Bcl-2 protein expression and downregulated Bax protein expression in pericytes (Figures 8(c) and $8(\mathrm{~d})$ ). The antiapoptotic effect of apelin was through increased expression of $\mathrm{Bcl}-2$ and reduced expression of Bax. In hypoxia group, the ratio of $\mathrm{Bcl}-2 / \mathrm{Bax}$ decreased $36 \%$ and LV-apelin group has a similar ratio $(P<0.001$, compared with con. group). However, the ratio of $\mathrm{Bcl}-2 / \mathrm{Bax}$ in treatment with apelin group was significantly increased $(P<0.001$, compared with hypoxia group) (Figures $8(\mathrm{e})$ and $8(\mathrm{f}))$.

\section{Discussion}

Apelin interacts with its specific receptor APJ, has multiple biological activities, and had been characterized in various tissues [31]. Previously, we proved that vitreous concentrations of apelin were significantly higher in the proliferative diabetic retinopathy (PDR) group. Likewise, apelin and APJ also colocalized with endothelial cells maker CD 31 in PDR [30]. In the present study, we further demonstrated that APJ was strong expressed in fibrovascular membranes of the PDR and was colocalized with pericytes. Therefore, apelin/APJ system was possibly involved in the pathological progression of PDR. However, the effect of apelin on apoptosis of primary retinal pericytes remains unknown. In order to study the effects of apelin in pericyte, primary rat pericytes were used here. Based on the current results, we proved the expression of apelin and APJ in pericytes and demonstrated that apelin and APJ are upregulated in hypoxia cultured condition. Knockdown of apelin inhibits proliferation and migration of pericytes. Moreover, exogenous recombinant apelin effectively prevented hypoxia-induced apoptosis through downregulating the expression of active-caspase 3 and increased the ratio of $\mathrm{Bcl}-2 / \mathrm{Bax}$ in pericyte. These results establish the foundation for further study of diseases associated with ischemia and hypoxia.

As we all know, rodent is similar to human in genetic background. In previous published studies, primary cultured pericytes were mostly originated from bovine retina, which restricted further in vivo studies [32]. In the present study, we established a rodent- (rat-) based primary pericyte in vitro model. By using a magnetic beads isolation method, we obtained primary rat retinal pericytes successfully in the purity of $90 \%$. As for marker of pericytes, alpha smooth muscle actin ( $\alpha$-SMA), tropomyosin desmin, nestin, sulfatide or nerve/glial antigen-2 (NG2) proteoglycan, platelet-derived growth factor receptor-B (PDGFR-B), aminopeptidase $\mathrm{N}$ (CD13), and the regulator of G-signaling 5 (RG5) are common pericyte markers $[4,33]$. However, no single entirely pericytespecific marker is known to date, and all markers currently used are dynamic in their expression and may be up- or downregulated in conjunction with developmental states, pathological reactions, and in vitro culturing conditions [4]. For example, pericytes on normal capillaries typically express desmin, but not SMA, whereas smooth muscle cells on arterioles and pericytes on venues are immunoreactive for both [34]. Therefore, we select three markers that sufficiently 


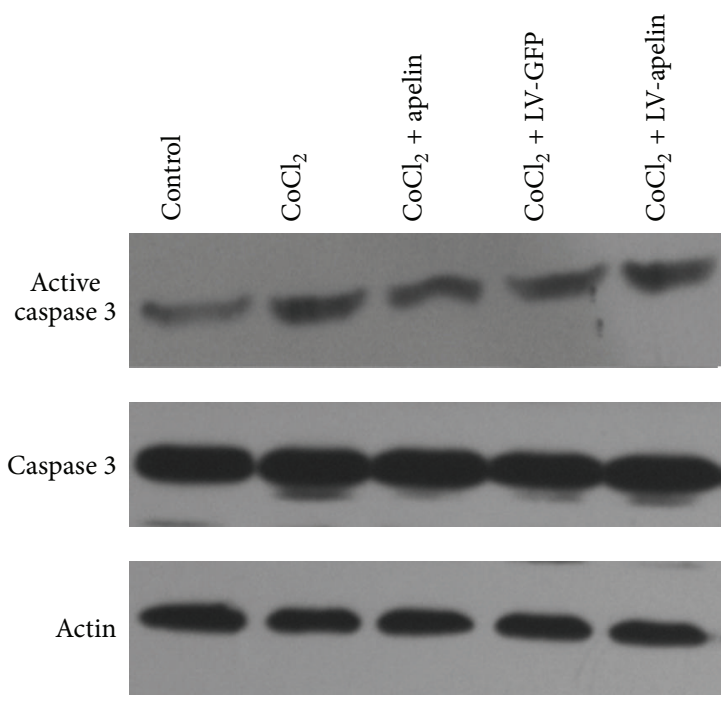

(a)
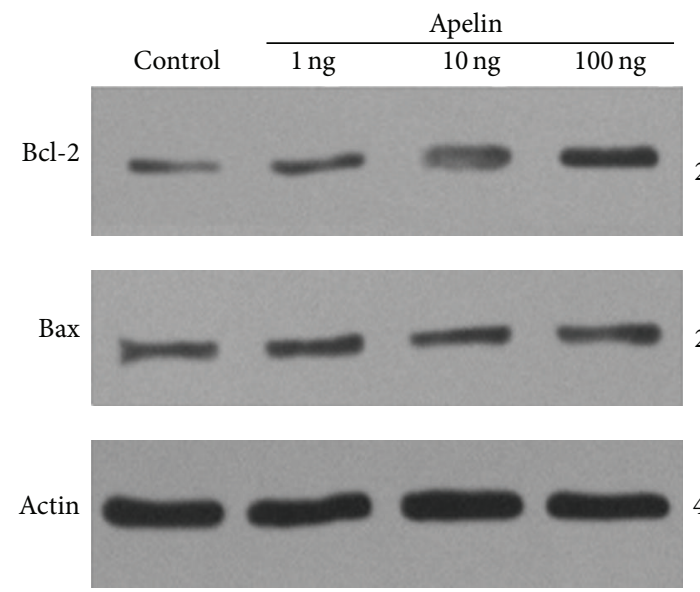

(c)
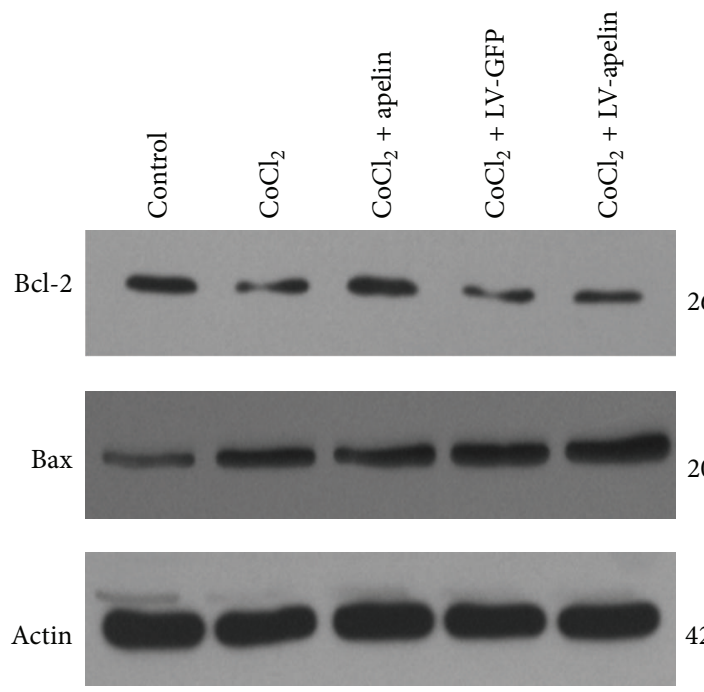

(e)

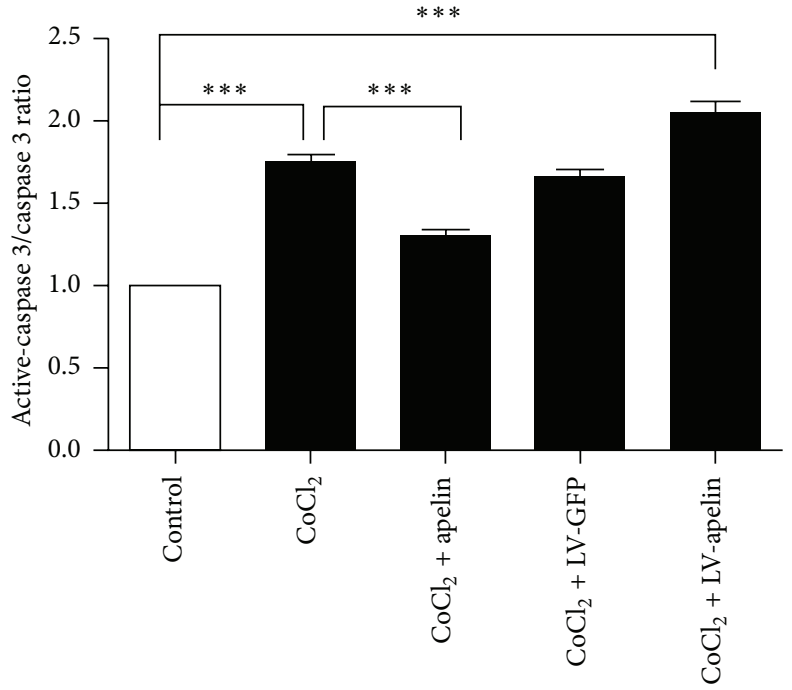

(b)

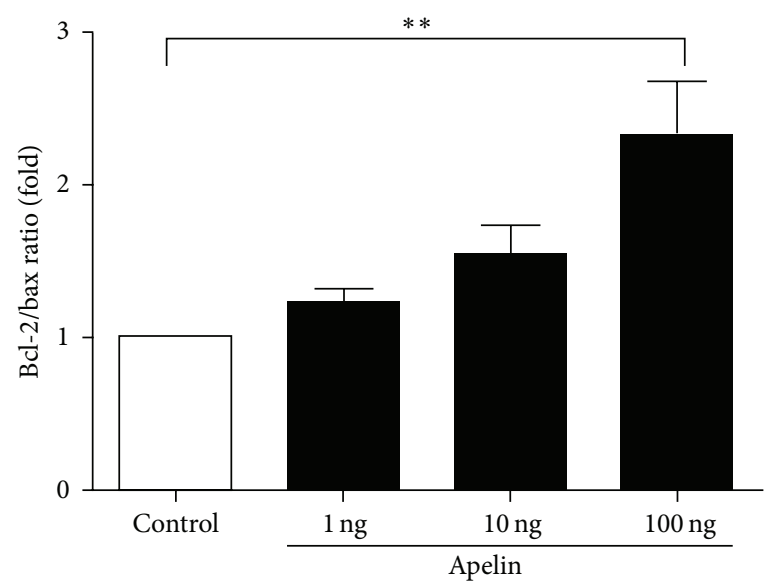

(d)

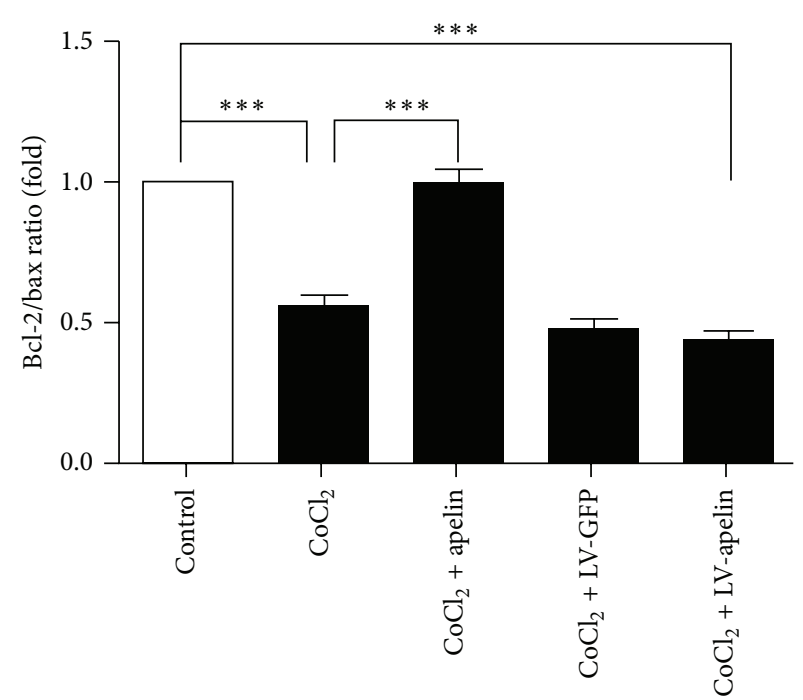

(f)

FIGURE 8: Effect of apelin on hypoxia-induced apoptosis in rat retinal pericytes. (a) Active-caspase 3 protein level significantly increased 12 h after hypoxia injury $(P<0.001)$. Apelin significantly decreased its levels after hypoxia injury $(P<0.01)$. (c and e) Effects of apelin on Bcl-2 and Bax protein expression in rat retinal pericytes. Cells were incubated with apelin and LV-apelin knockout under hypoxia. Western blot analysis was quantitated by densitometry of autoradiographs, and the relative mean ratio of Bcl-2/Bax was increased in apelin group $(P<0.001$ versus con.) and reduced in Lv-apelin knockdown group $(P<0.001$ versus con. $)$. 
identify pericytes in order to obtain highly pure rat retinal pericytes. In present study, pericytes uniformly expressed the cellular markers PDGFR- $\beta$, NG2 and desmin. Our results are consistent with Liu's study, who also proved these markers expressed in pericytes isolated from rats by mechanical morcellation and collagenase digestion [29].

Apelin/APJ is localized in a wide variety of tissues, including the endothelial cells of the primary blood vessels, neurons, and oligodendrocytes [18]. The lines of evidence show that apelin exerts its biological functions through its interaction with APJ. Knockout of apelin or APJ leads to the inhibition of both hypoxia-induced endothelial cell proliferation in vitro and hypoxia-induced vessel regeneration in the caudal fin regeneration of Fli-1 transgenic zebrafish [35]. Therefore, location of APJ in cells or tissues is very important with regard to apelin exerting its diverse functions. In the present study, through immunofluorescence staining, we first confirmed the expression of APJ in pericytes and hypoxia-induced upregulation of apelin and APJ. The results of this study showed that the expression of APJ was positive in pericytes, which is essential for apelin/APJ system and plays a role in pathological and physiological condition. This suggested that apelin might be involved in pericyte physiology and pathology.

Apelin was shown to have angiogenic activity in retinal endothelial cells, both in vitro and in vivo [18]. In our previous studies, we showed that apelin can enhance proliferation and migration of Müller cells and RPE cells [19, 21]. Eyries identified apelin as a hypoxia-inducible factor-1 (HIF-1) target gene and demonstrated that, under hypoxia, HIF-1 binds to the first intron of apelin, leading to upregulation of apelin expression [35]. In the present study, we observed that the viability and migration of pericytes incubated with various concentrations of apelin were enhanced. Under hypoxia exposure, pericytes viability decreased significantly with time-dependent manner and apelin can protect pericytes viability. Furthermore, knockdown of apelin led to a significant decrease in pericyte viability. These results further support the hypothesis that apelin is sensitive to hypoxia, playing a key role in hypoxia-induced pericyte proliferation and migration.

Many in vitro and in vivo insults, such as hypoxia and ischemia, trigger mixed cell death composed of both necrosis and apoptosis [31, 36]. Hypoxia-induced Bax upregulation, $\mathrm{Bcl}-2$ downregulation, and caspase 3 activation in variety of cells were reversed by HIF-1 overexpression and lead to the acquisition of antiapoptotic properties [37-39]. The ratio of antiapoptotic to proapoptotic proteins, especially the Bcl2/Bax ratio, determines susceptibility to apoptosis [40]. We therefore investigated whether these pathways were involved in the antiapoptotic effects of apelin in pericytes. Our result was consistent with previous studies, which indicated that $\mathrm{Bcl}-2 / \mathrm{Bax}$ apoptotic signaling pathways mediate the protective effects of the apelin/APJ system in vascular smooth muscle cells and osteoblasts [17, 24]. Caspases, cysteine proteases with aspartate specificity, are important mediators of apoptosis. Caspase 3 is effector caspase that is responsible for cleaving nucleases in addition to cellular substrates. We also revealed that apelin reduced caspase 3 activity, which suggests that apelin inhibits pericyte apoptosis through regulation of activity of caspase 3 and Bcl-2/Bax expression. Therefore, there is a growing consensus that apelin may be a promising therapeutic target against hypoxia/ischemia in the future.

In conclusion, this study demonstrated that apelin/APJ was expressed in PDR patient's membranes and in rat retinal pericytes. Apelin can protect pericytes against hypoxiainduced apoptosis through regulation of activation of caspase 3 and Bcl-2/Bax expression. These results indicated that apelin could be a potential therapeutic target for retinal angiogenic diseases.

\section{Conclusion}

Pericytes are a population of cells that are involved in normal vessel architecture and contraction and regulated blood flow. Hypoxia causes decreasing of pericytes viability in a time-dependent manner and induced pericytes apoptosis. However, apelin regulated function of pericytes under hypoxia inversely in a concentration-dependent manner and effectively prevented hypoxia-induced apoptosis through downregulating active-caspase 3 expression and increasing the ratio of $\mathrm{Bcl}-2 / \mathrm{Bax}$.

\section{Conflict of Interests}

The authors declare that there is no conflict of interests regarding the publication of this paper.

\section{Acknowledgments}

This work has been supported by the National Natural Science Foundation of China (no. 81271027), EFSD/CDS/Lilly Grant (nos. 90561 and 94410), the Program for New Century Excellent Talents in University (no. NCET-12-0010), and Fok Ying Tong Education Foundation (Hong Kong).

\section{References}

[1] M. Hellström, H. Gerhardt, M. Kalén et al., "Lack of pericytes leads to endothelial hyperplasia and abnormal vascular morphogenesis," The Journal of Cell Biology, vol. 153, no. 3, pp. 543$554,2001$.

[2] P. Lindahl, B. R. Johansson, P. Levéen, and C. Betsholtz, "Pericyte loss and microaneurysm formation in PDGF-B-deficient mice," Science, vol. 277, no. 5323, pp. 242-245, 1997.

[3] P. Levéen, M. Pekny, S. Gebre-Medhin, B. Swolin, E. Larsson, and C. Betsholtz, "Mice deficient for PDGF B show renal, cardiovascular, and hematological abnormalities," Genes \& Development, vol. 8, no. 16, pp. 1875-1887, 1994.

[4] A. Armulik, G. Genové, and C. Betsholtz, "Pericytes: developmental, physiological, and pathological perspectives, problems, and promises," Developmental Cell, vol. 21, no. 2, pp. 193-215, 2011.

[5] D. von Tell, A. Armulik, and C. Betsholtz, "Pericytes and vascular stability," Experimental Cell Research, vol. 312, no. 5, pp. 623-629, 2006. 
[6] A. P. Hall, "Review of the pericyte during angiogenesis and its role in cancer and diabetic retinopathy," Toxicologic Pathology, vol. 34, no. 6, pp. 763-775, 2006.

[7] H.-P. Hammes, J. Lin, O. Renner et al., "Pericytes and the pathogenesis of diabetic retinopathy," Diabetes, vol. 51, no. 10, pp. 3107-3112, 2002.

[8] Y. Feng, F. vom Hagen, F. Pfister et al., "Impaired pericyte recruitment and abnormal retinal angiogenesis as a result of angiopoietin-2 overexpression," Thrombosis and Haemostasis, vol. 97, no. 1, pp. 99-108, 2007.

[9] S. Hughes, T. Gardiner, L. Baxter, and T. Chan-Ling, "Changes in pericytes and smooth muscle cells in the kitten model of retinopathy of prematurity: Implications for plus disease," Investigative Ophthalmology and Visual Science, vol. 48, no. 3, pp. 1368-1379, 2007.

[10] Y. Kawamata, Y. Habata, S. Fukusumi et al., "Molecular properties of apelin: tissue distribution and receptor binding," Biochimica et Biophysica Acta-Molecular Cell Research, vol. 1538, no. 2-3, pp. 162-171, 2001.

[11] M. de Falco, L. de Luca, N. Onori et al., "Apelin expression in normal human tissues," In Vivo, vol. 16, no. 5, pp. 333-336, 2002.

[12] A. Reaux, K. Gallatz, M. Palkovits, and C. Llorens-Cortes, "Distribution of apelin-synthesizing neurons in the adult rat brain," Neuroscience, vol. 113, no. 3, pp. 653-662, 2002.

[13] H. Kidoya, M. Ueno, Y. Yamada et al., "Spatial and temporal role of the apelin/APJ system in the caliber size regulation of blood vessels during angiogenesis," The EMBO Journal, vol. 27, no. 3, pp. 522-534, 2008.

[14] C. M. Cox, S. L. D’Agostino, M. K. Miller, R. L. Heimark, and P. A. Krieg, "Apelin, the ligand for the endothelial Gprotein-coupled receptor, APJ, is a potent angiogenic factor required for normal vascular development of the frog embryo," Developmental Biology, vol. 296, no. 1, pp. 177-189, 2006.

[15] C. Hara, A. Kasai, F. Gomi et al., "Laser-induced choroidal neovascularization in mice attenuated by deficiency in the apelin-APJ system," Investigative Ophthalmology and Visual Science, vol. 54, no. 6, pp. 4321-4329, 2013.

[16] Q.-F. Liu, H.-W. Yu, L. You, M.-X. Liu, K.-Y. Li, and G.-Z. Tao, "Apelin-13-induced proliferation and migration induced of rat vascular smooth muscle cells is mediated by the upregulation of Egr-1," Biochemical and Biophysical Research Communications, vol. 439, no. 2, pp. 235-240, 2013.

[17] R.-R. Cui, D.-A. Mao, L. Yi et al., "Apelin suppresses apoptosis of human vascular smooth muscle cells via APJ/PI3-K/Akt signaling pathways," Amino Acids, vol. 39, no. 5, pp. 1193-1200, 2010.

[18] A. Kasai, N. Shintani, M. Oda et al., "Apelin is a novel angiogenic factor in retinal endothelial cells," Biochemical and Biophysical Research Communications, vol. 325, no. 2, pp. 395-400, 2004.

[19] D. Qin, X.-X. Zheng, and Y.-R. Jiang, "Apelin-13 induces proliferation, migration, and collagen I mRNA expression in human RPE cells via PI3K/Akt and MEK/Erk signaling pathways," Molecular Vision, vol. 19, pp. 2227-2236, 2013.

[20] Q. Lu, Y.-R. Jiang, J. Qian, and Y. Tao, "Apelin-13 regulates proliferation, migration and survival of retinal Müller cells under hypoxia," Diabetes Research and Clinical Practice, vol. 99, no. 2, pp. 158-167, 2013.

[21] X.-L. Wang, Y. Tao, Q. Lu, and Y.-R. Jiang, “Apelin supports primary rat retinal Müller cells under chemical hypoxia and glucose deprivation," Peptides, vol. 33, no. 2, pp. 298-306, 2012.

[22] A. Sendoel and M. O. Hengartner, "Apoptotic cell death under hypoxia," Physiology, vol. 29, no. 3, pp. 168-176, 2014.
[23] Y. Fang, Q. Zhang, J. Tan, L. Li, X. An, and P. Lei, "Intermittent hypoxia-induced rat pancreatic beta-cell apoptosis and protective effects of antioxidant intervention," Nutrition \& Diabetes, vol. 4, no. 9, article e131, 2014.

[24] H. Xie, L.-Q. Yuan, X.-H. Luo et al., "Apelin suppresses apoptosis of human osteoblasts," Apoptosis, vol. 12, no. 1, pp. 247-254, 2007.

[25] S.-Y. Tang, H. Xie, L.-Q. Yuan et al., "Apelin stimulates proliferation and suppresses apoptosis of mouse osteoblastic cell line MC3T3-E1 via JNK and PI3-K/Akt signaling pathways," Peptides, vol. 28, no. 3, pp. 708-718, 2007.

[26] X. Zeng, S. P. Yu, T. Taylor, M. Ogle, and L. Wei, "Protective effect of apelin on cultured rat bone marrow mesenchymal stem cells against apoptosis," Stem Cell Research, vol. 8, no. 3, pp. 357367, 2012.

[27] O. S. Kim, J. Kim, C.-S. Kim, N. H. Kim, and J. S. Kim, “KIOM79 prevents methyglyoxal-induced retinal pericyte apoptosis in vitro and in vivo," Journal of Ethnopharmacology, vol. 129, no. 3, pp. 285-292, 2010.

[28] J. Cai, O. Kehoe, G. M. Smith, P. Hykin, and M. E. Boulton, "The angiopoietin/Tie-2 system regulates pericyte survival and recruitment in diabetic retinopathy," Investigative Ophthalmology and Visual Science, vol. 49, no. 5, pp. 2163-2171, 2008.

[29] G. Liu, C. Meng, M. Pan et al., "Isolation, purification, and cultivation of primary retinal microvascular pericytes: a novel model using rats," Microcirculation, vol. 21, no. 6, pp. 478-489, 2014.

[30] Y. Tao, Q. Lu, Y.-R. Jiang et al., “Apelin in plasma and vitreous and in fibrovascular retinal membranes of patients with proliferative diabetic retinopathy," Investigative Ophthalmology \& Visual Science, vol. 51, no. 8, pp. 4237-4242, 2010.

[31] A. Y. Xiao, L. Wei, S. Xia, S. Rothman, and S. P. Yu, "Ionic mechanism of ouabain-induced concurrent apoptosis and necrosis in individual cultured cortical neurons," The Journal of Neuroscience, vol. 22, no. 4, pp. 1350-1362, 2002.

[32] E. Beltramo, E. Berrone, S. Giunti, G. Gruden, P. C. Perin, and M. Porta, "Effects of mechanical stress and high glucose on pericyte proliferation, apoptosis and contractile phenotype," Experimental Eye Research, vol. 83, no. 4, pp. 989-994, 2006.

[33] M. Krueger and I. Bechmann, "CNS pericytes: concepts, misconceptions, and a way out," Glia, vol. 58, no. 1, pp. 1-10, 2010.

[34] S. Morikawa, P. Baluk, T. Kaidoh, A. Haskell, R. K. Jain, and D. M. McDonald, "Abnormalities in pericytes on blood vessels and endothelial sprouts in tumors," The American Journal of Pathology, vol. 160, no. 3, pp. 985-1000, 2002.

[35] M. Eyries, G. Siegfried, M. Ciumas et al., "Hypoxia-induced apelin expression regulates endothelial cell proliferation and regenerative angiogenesis," Circulation Research, vol. 103, no. 4, pp. 432-440, 2008.

[36] L. Wei, D. J. Ying, L. Cui, J. Langsdorf, and S. Ping Yu, "Necrosis, apoptosis and hybrid death in the cortex and thalamus after barrel cortex ischemia in rats," Brain Research, vol. 1022, no. 1-2, pp. 54-61, 2004.

[37] B. Yang, K. He, F. Zheng et al., "Over-expression of hypoxiainducible factor-1 alpha in vitro protects the cardiac fibroblasts from hypoxia-induced apoptosis," Journal of Cardiovascular Medicine, vol. 15, no. 7, pp. 579-586, 2014.

[38] B.-F. Wang, X.-J. Wang, H.-F. Kang et al., "Saikosaponin-D enhances radiosensitivity of hepatoma cells under hypoxic conditions by inhibiting hypoxia-inducible factor-lalpha," Cellular Physiology and Biochemistry, vol. 33, no. 1, pp. 37-51, 2014. 
[39] A. Nishimoto, N. Kugimiya, T. Hosoyama, T. Enoki, T.-S. Li, and K. Hamano, "HIF-1 $\alpha$ activation under glucose deprivation plays a central role in the acquisition of anti-apoptosis in human colon cancer cells," International Journal of Oncology, vol. 45, no. 6, pp. 2077-2084, 2014.

[40] Z. N. Oltvai, C. L. Milliman, and S. J. Korsmeyer, "Bcl-2 heterodimerizes in vivo with a conserved homolog, Bax, that accelerates programed cell death," Cell, vol. 74, no. 4, pp. 609619, 1993. 


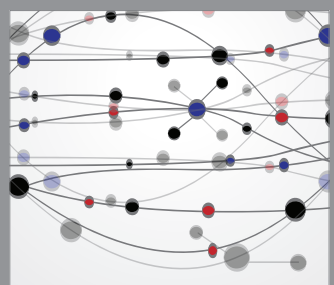

The Scientific World Journal
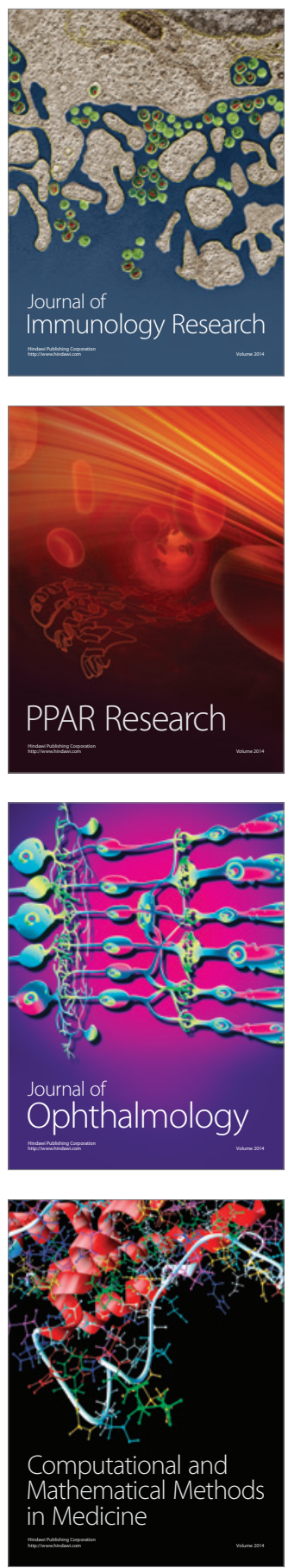

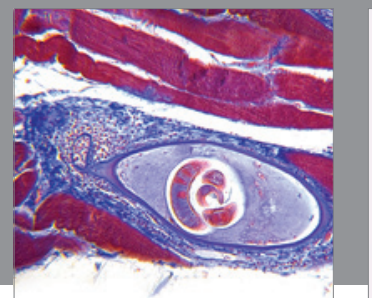

Gastroenterology

Research and Practice
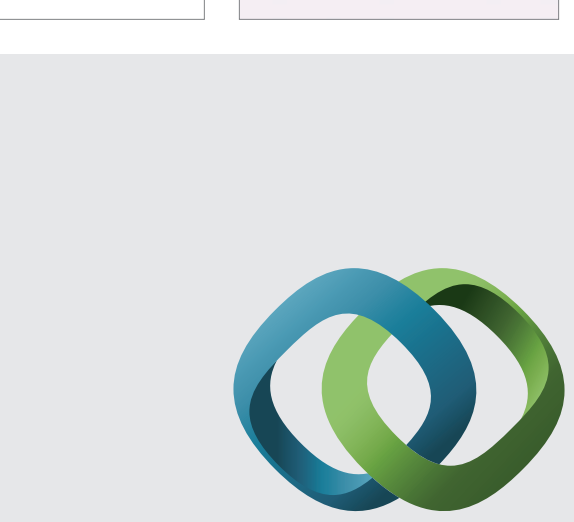

\section{Hindawi}

Submit your manuscripts at

http://www.hindawi.com
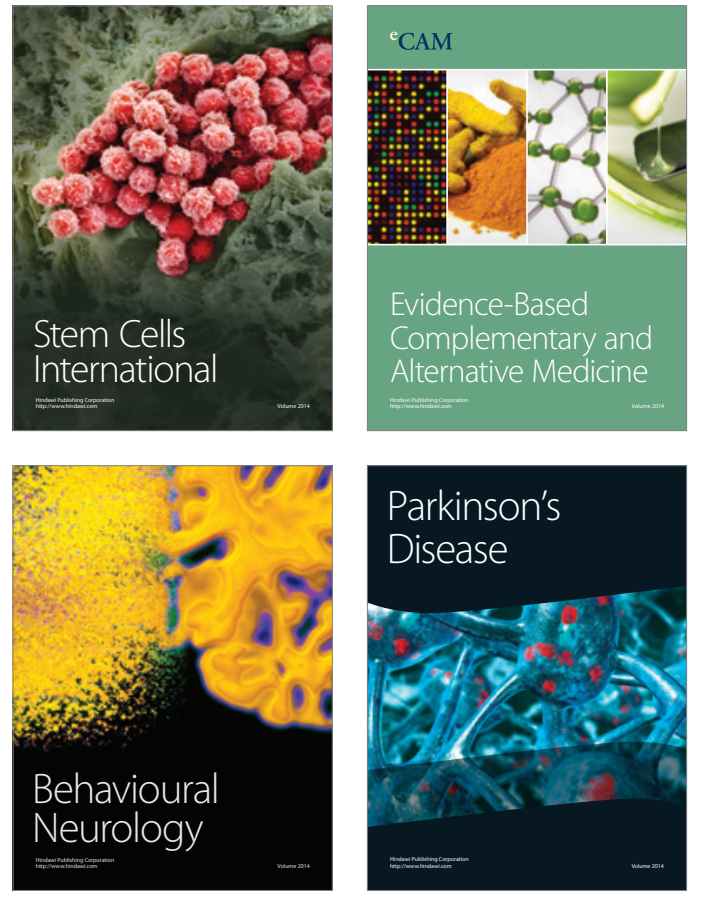
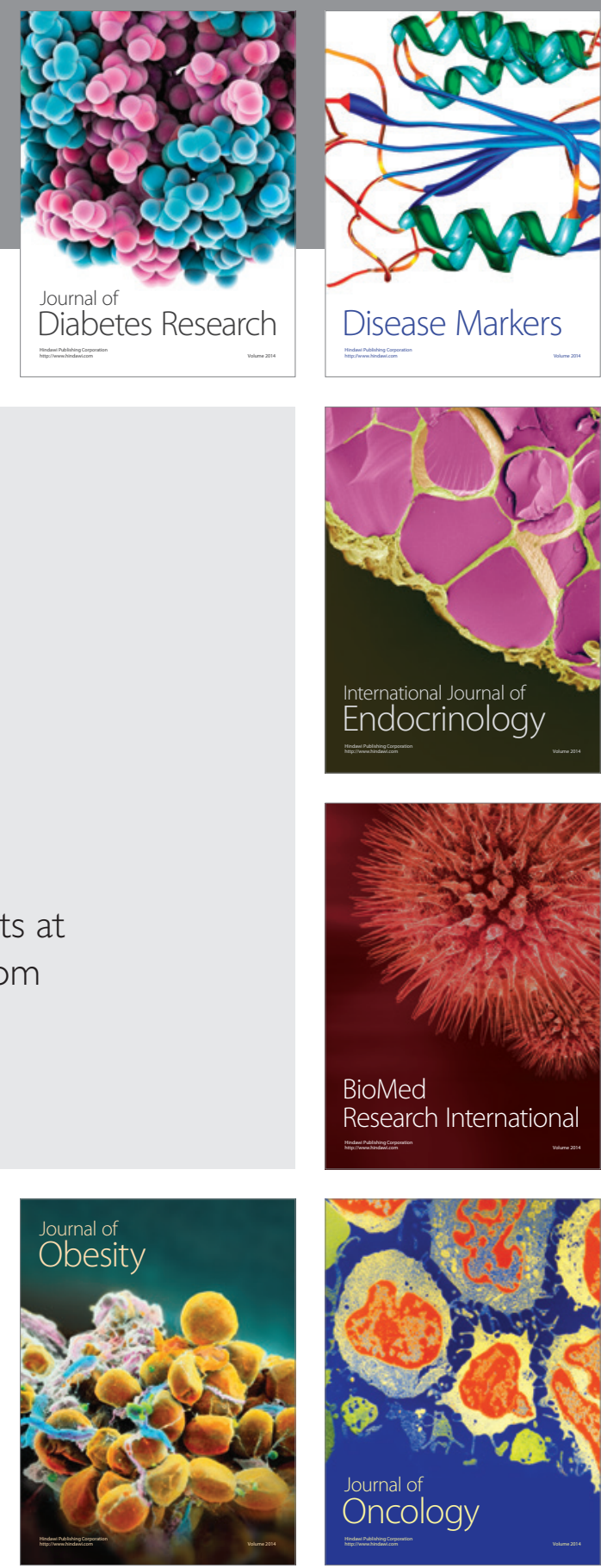

Disease Markers
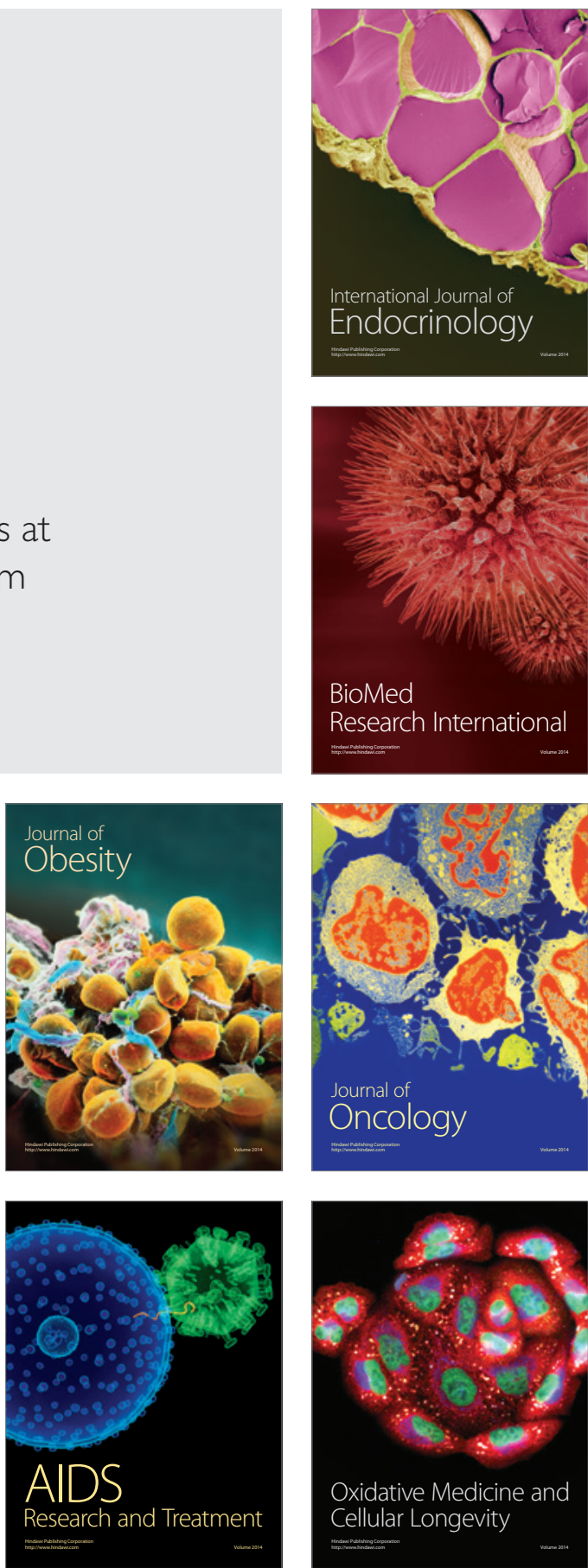